\title{
Astrocytes \& Astrocyte derived Extracellular Vesicles in Morphine Induced Amyloidopathy: Implications for Cognitive Deficits in Opiate Abusers
}

\author{
Susmita Sil*, Seema Singh, Divya T. Chemparathy, Ernest T. Chivero, Lila Gordon, Shilpa Buch* \\ Department of Pharmacology and Experimental Neuroscience, University of Nebraska Medical Center, Omaha, \\ NE 68198-5880, USA.
}

[Received February 16, 2021; Revised April 5, 2021; Accepted April 6, 2021]

\begin{abstract}
While opiates like morphine play a major role in the pharmacotherapy for the control of pain associated with various diseases, paradoxically, their long-term use is associated with cognitive impairments. Furthermore, morphine administration has been shown to result in neuronal synaptodendritic injury in rodent brains, leading to neurodegeneration. We recently reported the role of astrocytes as contributors of amyloidosis associated with HIV-associated neurological disorders. Herein we hypothesize that morphine could induce astrocytic amyloidosis, which, in turn, could be disseminated to various regions in the brain by astrocyte-derived EVs (ADEVs). In this study we demonstrate brain region-specific up-regulation of astrocytic amyloids in morphine dependendent rhesus macaques. In addition, herein we also demonstrate increased expression of $\beta$-site cleaving enzyme (BACE1), APP, and A $\beta$ in human primary astrocytes (HPAs) exposed to morphine. Mechanisms

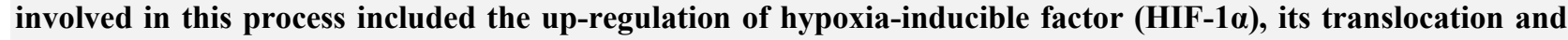
binding to the promoter of BACE1, leading to increased BACE1 activity and, generation of A $\beta$ 1-42. Gene silencing approaches confirmed the regulatory role of HIF-1 $\alpha$ in BACE1 mediated amyloidosis leading to astrocyte activation and neuroinflammation. We next sought to assess whether morphine-stimulated ADEVs could carry amyloid cargoes. Results showed that morphine exposure induced the release of morphine-ADEVs, carrying amyloids. Interestingly, silencing HIF-1 $\alpha$ in astrocytes not only reduced the numbers of ADEV released, but also the packaging of amyloid cargos in the ADEVs. These findings were further validated in brain derived EVs (BEVs) isolated from macaques, wherein it was shown that BEVs from morphine-dependent macaques, carried varieties of amyloid cargoes including the cytokine IL-1ß. This is the first report implicating the role of HIF-1 $\alpha$-BACE1 axis in morphine-mediated induction of astrocytic amyloidosis, leading, in turn, to neuroinflammation and release of the amyloid cargoes via ADEVs. These findings set the groundwork for the future development of therapeutic strategies for targeting cognitive deficits in chronic opiate users.
\end{abstract}

Key words: Morphine, astrocytes, amyloids, EVs, HIF-1 $\alpha$

Morphine is one of the most potent prescription analgesics among all the opiates that is used extensively in the clinical setting [1, 2]. On, an average $17 \%$ or more of Americans have at least one opioid prescription filled, with a mean of 3.4 opioid prescriptions dispensed per patient (www.cdc.gov/drugoverdose/data/prescribing/ prescribing-practices.html). As per the CDC, in 2017, there were 58 opioid prescriptions written for every 100 Americans. Infact, from 2007 to 2017, there has been 2.6-fold increase in opioid overdose deaths (NIH, 2019). Several reports have shown that extensive opioid use leads to complications including addiction, tolerance, cognitive impairment, withdrawal symptoms, memory impairment, depression and severely compromised

*Correspondence should be addressed to: Dr. Susmita Sil (Email: susmita.sil@unmc.edu) and Dr. Shilpa Buch (Email: sbuch@unmc.edu), University of Nebraska Medical Center, Omaha, NE 68198-5880, USA.

Copyright: ( $92021 \mathrm{Li} \mathrm{CH}$ et al. This is an open-access article distributed under the terms of the Creative Commons Attribution License, which permits unrestricted use, distribution, and reproduction in any medium, provided the original author and source are credited. 
immune system as well as increased risk of opportunistic infections [3-12]. Furthermore, it has also been reported that patients undergoing short-term hospital stay for surgery are also at an increased risk of long-term dependence on prescription opioids [13]. With the increased morbidity associated with pain-related conditions such as cancer and neuropathies, morphine abuse has escalated globally and has become a serious global problem, leading, in turn, to comorbidity of opioid induced cognitive dysfunction [14]. Approximately, 20$44 \%$ of the cancer patients taking morphine develop cognitive decline [14]. In keeping with this, other reports have also shown impairment of prospective memory [12], as well as attention, complex working memory, and episodic memory $[15,16]$ in long-term opiate users. Emerging evidence suggests that neural circuits underlying opiate use disdorders share some common pathways as those underlying learning and memory.

Alzheimer's Disease (AD) is a dreaded neurodegenerative disease characterized by the presence of extracellular amyloid plaques and intraneuronal tangles $[17,18]$, which play a key role in neurodegeneration \& cognitive impairment. Amyloid $\beta(A \beta)$ is a key molecule implicated in the development of AD pathogenesis [19], which is generated from sequential cleavage of the amyloid precursor protein (APP) by two proteolytic enzymes- $\beta$ - secretase (beta-site APP cleavage enzyme, BACE) and $\gamma$-secretase $[20,21]$. Dysregulation of the BACE1 enzyme and/ or $\gamma$-secretase leads to the production and accumulation of $A \beta$, thereby contributing to the pathogenesis of $\mathrm{AD}[22,23]$. In addition to amyloids [24], there are also other reports demonstrating increased deposition of hyperphosphorylated tau in the brain regions (frontal, temporal cortex and locus coeruleus) of opiate users compared to age matched controls $[24,25]$. It is likely that use of opiates such as morphine, that has detrimental effects on cognitive functioning, could likely attribute to $\mathrm{AD}$-like brain pathology. There are no reports on this phenomenon and warrant further investigation.

In our recent report we showed that HIV-1 protein Tat induced astrocytic amyloidosis involving the HIF-1 $\alpha$ BACE1-AS axis, leading to the development of AD- like pathology in both HIV-1+ patients and in SIV-infected macaques, that was linked with HIV-associated neurological disorders (HAND) [26]. Based on the premise that HIV-infected patients are also afflicted with substance abuse disorders (SUDs), specifically opiates, we wanted to test whether opiates on their own could also mediate cognitive deficits and if so, what were the mechanisms involved. Furthermore, studies from our group have demonstrated earlier that morphine exposure mediates neuronal synaptodendritic injury in neuronal cultures as well as rodent brains involving the endoplasmic reticulum (ER) stress-autophagy axis, and leading to neurodegeneration [27]. Additionally, several reports have also shown morphine mediated cognitive dysfunctioning both in rodents as well as in patients on opiate therapy $[11,12]$. In AD, it is suggested that one of the key causes of cognitive impairment is dysregulation and accumulation of $A \beta$, happening early in the disease and often preceeding the onset of dementia. More recently, in addition to neurons as the source of $A \beta$, other cells such as the astrocytes that comprise $\sim 50-70 \%$ of the total cell population in the central nervous system (CNS), have also garnered interest as potential contributors of amyloidosis in $\mathrm{AD}[28,29]$. If indeed these cells also play a role in inducing amyloidosis, this process could translate into a significant added burden to the process of brain amyloidosis. Till date, however, there are no reports on the role of morphine in astrocytic amyloidosis, leading to neuroinflammation which, in turn, could manifest as cognitive impairments.

Recent studies have also shown that endosomederived multivesicular bodies can function as conduits for transferring intercellular cargoes to extracellular space (ECS) and to neighboring or distant recipient cells [30]. Extracellular Vesicles (EVs) play important roles as cargo-carrying vesicles mediating communication among diverse cell types and tissues [31-33]. Furthermore, the release kinetics of EVs have also shown to be altered in various pathologies [34-38]. EVs have been implicated to play key roles in various neurodegenerative diseases, such as Alzheimer's Disease [39], Parkinson's disease [40], Amyotrophic Lateral Sclerosis [41] as well as HIVassociated neurocognitive disorders (HAND) [42]. Additionally, in both homeostatis as well as disease state astrocyte derived EVs (ADEVs) have been shown to play critical roles in modulating cellular functions in the CNS $[43,44]$. In AD, EVs carrying amyloid cargoes have been postulated to serve as biomarkers of disease pathogenesis [45] while also playing a role in seeding of amyloids, thus disseminating the disease [46], leading to disease progression and severity [47-49].

Taken together we thus hypothesized that morphine could induce the process of astrocytic amyloidosis leading to neuroinflammation, and these amyloids could be packaged in the EVs, leading to their dissemination in brain areas, thereby correlating with cognitive deficits. In the current study, we report astrocytic amyloidosis in the archival brain tissue of rhesus macaques chronically administered morphine. We also report that the neurotoxic amyloid cargoes were packaged in the brain-derived EVs from macaque brains. Furthermore, dissection of the molecular pathway underlying morphine-mediated induction of amyloids in human primary astrocytes (HPAs) involved up-regulated expression of HIF-1 $\alpha$ and its regulation of the BACE1 promoter, leading to 
increased expression of the cleaved toxic $A \beta$ 1-42 form, ultimately resulting in astrogliosis and neuroinflammation. Furthermore, we found that these amyloids and neuroinflammatory cargoes were packaged in the ADEVs and released to the ECS, which could contribute to neurodegeneration and neuroinflammation involving the HIF- $1 \alpha$-BACE1 axis. This is the first report describing morphine-mediated induction of astrocytic amyloidosis $\&$ its release in EVs involving the HIF-1 $\alpha$-BACE1 axis. HIF- $1 \alpha$ can thus be considered as a potential therapeutic target for ameliorating morphine-induced cognitive impairment in patients on chronic opiate therapy.

\section{MATERIALS AND METHOD}

\section{Reagents}

Following antibodies were used for this study. A $\beta$ mOC64 (ab201060, Abcam, MA, USA), A $\beta$ 1-42 (ab10148,), BACE1 (ab63954, Abcam, MA, USA), APP (ab15272, Abcam, MA, USA) CD63 (ab216130, Abcam, MA, USA), CD9 (ab92726, Abcam, MA, USA), Flotillin (ab133497, Abcam, MA, USA), TSG101 (ab125011, Abcam, MA, USA), Alix (ab275377, Abcam, MA, USA), Calnexin (ab133615, Abcam, MA, USA), Argonuate-2 (ab186733, Abcam, MA, USA), GFAP (G3893, SigmaAldrich, MO, USA), $\beta$-actin (A5316, Sigma- Aldrich, MO, USA), HIF-1 $\alpha$ (NB100-449, Novus Biological Company, CO, USA), goat anti-rabbit (sc-2004, Santa Cruz Biotechnology, TX, USA) and goat anti-mouse (sc2005, Santa Cruz Biotechnology, TX, USA), Alexa Fluor 488 conjugated goat anti-mouse (A11001, ThermoFisher Scientific, MA, USA), Alexa Fluor 647 conjugated goat anti-mouse (A32728, ThermoFisher Scientific, MA, USA), Alexa Fluor 594 conjugated goat anti-rabbit (A11012, ThermoFisher Scientific, MA, USA).

\section{Macaques}

Archival brain tissues from Indian rhesus macaques (Macaca mulatta) were used for this study. All the macaque experiments were conducted according to the protocols approved by the Institutional Animal Care and Use Committee. Macaques were exposed to morphine as described previously [50]. Briefly, 12 weeks of morphine injections (i.m., three times daily) were administered in monkeys $(\mathrm{n}=4)$ with an initial dose of $6 \mathrm{mg} / \mathrm{kg}$ body weight (b.w.) in the first week, $9 \mathrm{mg} / \mathrm{kg}$ b.w. in the second week followed by $12 \mathrm{mg} / \mathrm{kg}$ b.w. for the remaining 10 weeks. For the control group, monkeys $(n=4)$ received saline three times daily for 12 weeks. At the end of the treatment period of 12 weeks, the animals were sacrificed, and different brain regions were dissected and processed for the further experiments. Brain homogenates were used for assessing the expression levels of various proteins and mRNAs of interest as well as EV isolation. Paraffin fixed sections were used to determine the expression levels of $\mathrm{A} \beta$ 1-42, $\mathrm{A} \beta$ m0C64 and GFAP immunostaining.

\section{Cell culture}

Human primary astrocytes (HPA) were procured from ScienCell Research Laboratories (1800, Carlsbad, USA) and were grown in astrocyte culture media (1801, ScienCell Research Laboratories, Carlsbad, CA) supplemented with $2 \%$ fetal bovine serum (0010, ScienCell Research Laboratories, Carlsbad, CA), astrocyte growth supplement (1852, ScienCell Research Laboratories, Carlsbad, CA) and penicillin-streptomycin solution (0503, ScienCell Research Laboratories, Carlsbad, CA) until $70 \%$ confluency at $37^{\circ} \mathrm{C}$ in a humidified incubator with $5 \% \mathrm{CO}_{2}$. HPAs were cultured at a density of $0.3 \times 10^{6} /$ well ( 6 well plate) and $0.05 \times$ $10^{6} /$ well (24 well plate) for the experiments with a maximum of 5 passages. Cells were serum-starved prior to exposure to morphine.

\section{Quantitative real-time PCR (qPCR)}

Total RNA was isolated from HPAs using Quick-RNA MicroPrep kit (Zymo Research Corporation, USA, R1055) according to the manufacturer's instruction. qPCR was performed as described previously [51]. Briefly, $1 \mu \mathrm{g}$ of total RNA was reversely transcribed into complementary DNA using Verso cDNA synthesis kit (Thermo Fisher Scientific, USA, AB1453/B) as per the manufacturer's protocol. qPCR reactions were performed using Taqman probes. The commercial primers for BACE1 (Hs_01121195), APP (Hs_00169098), HIF-1 $\alpha$ (Hs_00153153), GAPDH (Hs_002786624), TNF- $\alpha$ (Hs_00174128), IL-1 $\beta$ (Hs_01555410), IL-6 (Hs_00 174131) were purchased from Thermo Fisher Scientific, USA. Relative values were obtained by normalizing averaged CT values to housekeeping control GAPDH and the fold change in the gene expression was calculated by $2^{-\Delta \Delta C T}$ method.

\section{Western blotting}

Standard western blotting procedure was followed to analyze the protein expressions in samples as described previously [26]. Briefly, proteins were extracted from HPAs as well as brain tissues of experimental macaques using the Mammalian Cell Lysis kit (MCL1-1KT, SigmaAldrich, St. Louis, MO, USA) and were quantified by BCA assay using Pierce BCA Protein Assay Kit (23227, Thermo Fisher Scientific, USA) according to the manufacturer's instruction. The proteins were separated 
by SDS PAGE and were transferred to PVDF membrane (IPVH00010, Millipore Sigma, MO, USA). Immunoblotting was performed using specific primary antibodies and horseradish peroxidase linked anti-mouse and antirabbit IgGs. The immunocomplex was detected by SuperSignal chemiluminescent substrate (VJ311133, Thermo Fisher Scientific, USA) according to the manufacturer's instruction. $\beta$-actin antibody was used to normalize the protein expression. Images of protein bands were acquired using a digital photo scanner GT-X750 (Seiko Epson Corp) and were quantified using Image $\mathbf{J}$ (v1.4.3.67; NIH, Bethesda, MD) software.

\section{siRNA Transfection}

siRNA transfection has been carried out as described previously [52]. In brief, HPAs were seeded in 6-well plates $\left(0.3 \times 10^{6}\right.$ cells per well $)$ and thereafter, incubated overnight at $37^{\circ} \mathrm{C}$ in a humidified, $5 \% \mathrm{CO}_{2}$ incubator. Human HIF-l $\alpha$ siRNA (sc-35561, Santa Cruz Biotechnology, TX, USA) and human BACE1 siRNA (sc37224, Santa Cruz Biotechnology, TX, USA) were used to silence the expressions of HIF-1 $\alpha$ and BACE1 expressions respectively in HPAs $\left(0.3 \times 10^{6}\right.$ cells per well). Gene silencing was confirmed by qPCR and Western blotting.

\section{B secretase activity}

HPAs $\left(0.3 \times 10^{6}\right.$ cells $)$ at $70 \%$ confluency were exposed with morphine $(0.5 \mu \mathrm{M})$ for $24 \mathrm{~h}$. After the experimental period, the cells were washed in ice-cold PBS and BACE1 activity was assessed using fluorometric based BetaSecretase Activity Assay Kit (ab65357, Abcam, MA, USA) according to the manufacturer's protocol.

\section{Immunohistochemistry}

Formaldehyde fixed paraffin-embedded tissue sections (5 $\mu \mathrm{m})$ of frontal cortex and basal ganglia of saline and morphine treated macaques were deparaffinized in xylene and re-hydrated in descending grades of ethanol and deionized water. Following antigen retrieval in Tris EDTA buffer ( $\mathrm{pH}, 9.0)$ and blocking, the tissue sections were probed with primary antibodies A $\beta$ 1-42, A $\beta$ m0C64 and GFAP. Immunostained tissues were detected with respective fluorescent-conjugated secondary antibodies and the images were acquired using Z1 inverted microscope (Carl Zeiss, Thornwood, NY). All the images were analyzed using the AxioVs 40 Version 4.8.0.0 software (Carl Zeiss MicroImaging $\mathrm{GmbH}$ ) and quantified by Image J (v1.4.3.67; NIH, Bethesda, MD) software.

\section{Isolation of EVs from astrocytes}

Extracellular vesicles (EVs) were isolated from the cell culture supernatant of HPAs by differential centrifugations as described previously [43]. Briefly, conditioned media were harvested from different treatment groups of HPAs and, centrifuged at $300 \times \mathrm{g}$ for $10 \mathrm{~min}$ and $2000 \times \mathrm{g}$ for $10 \mathrm{~min}$ to remove cell debris. The supernatants were centrifuged again at $10,000 \times \mathrm{g}$ for 30 min and filtered through $0.22-\mu \mathrm{m}$ filter. Exosomes were concentrated by ultracentrifugation at $100,000 \times \mathrm{g}$ for 70 min (Beckman Ti32 rotor; Beckman Coulter, Brea, CA, USA) and exomeres were concentrated by ultracentrifugation at $167,000 \times \mathrm{g}$ for $16 \mathrm{~h}$ [53]. EVs were quantified by NTA analysis using Nanosight NS300 instrument as described previously $[43,54]$. EVs were further characterized for the expression of fraction specific markers by Western blotting, atomic force microscopy and transmission electron microscopy.

\section{Isolation of EVs from macaque brain}

For the isolation of EVs from macaque brain, saline and morphine administered macaque brains tissues (1000 mg) were homogenized in Hibernate A medium (10-15ml) containing proteolytic enzyme papain (20 units/mL) for $30 \mathrm{~min}$ on gentleMACS Octo Dissociator with heaters (Miltenyi Biotec, USA). Tissue homogenates were centrifuged at $300 \mathrm{x} \mathrm{g}$ for $10 \mathrm{~min}, 2000 \mathrm{x} \mathrm{g}$ for $10 \mathrm{~min}$ at $4^{\circ} \mathrm{C}$ to eliminate cell debris. Supernatants were collected and centrifuged at $10,000 \mathrm{x}$ g for $30 \mathrm{~min}$ to separate large vesicles. Supernatants were filtered through $0.2 \mu \mathrm{m}$ filters and the small particle sized EVs were collected through ultracentrifugation at $100,000 \mathrm{x}$ g for $70 \mathrm{~min}$ at $4^{\circ} \mathrm{C}$. EV pellets were further processed for gradient purification using 5, 10, 20 and 40\% iodixanol (Optiprep; Cosmo Bio USA) gradient as described previously [55]. Briefly, gradient was prepared by adding $40 \%$ iodixanol $(3 \mathrm{ml})$ at the bottom of the ultracentrifugation tube followed by layers of 20,10 and $5 \%$ of iodixanol $(3 \mathrm{ml} \mathrm{each})$ to the top. EV pellets were suspended in $500 \mu \mathrm{l}$ of $0.25 \mathrm{M}$ sucrose buffer [10 mM Tris, $\mathrm{pH}$ 7.4] and were added on the top of the layered iodixanol followed by ultracentrifugation at $100,000 \times \mathrm{g}$ in a SW40 Ti rotor for $18 \mathrm{~h}$ at $4^{\circ} \mathrm{C}$. After centrifugation, $500 \mu \mathrm{l}$ was discarded from the top and $1 \mathrm{ml}$ fractions $(\mathrm{F} 1-\mathrm{F} 12)$ were collected containing EVs into different tubes. The EVs were further washed with filtered PBS to remove iodixanol from the samples by ultracentrifugation at $200,000 \times \mathrm{g}$ in a SW40 Ti rotor for $2 \mathrm{~h}$ at $4^{\circ} \mathrm{C}$. EVs were quantified by NTA analysis using Nanosight NS300 instrument as described previously $[43,54]$. EVs fractions were further characterized for the expression of fraction specific 
markers by western blotting, atomic force microscopy and transmission electron microscopy.

\section{Zeta view tracking analysis}

Isolated EVs from the brain and HPAs were analyzed by nanoparticle tracking analysis (NTA) using ZetaView Nanoparticle Tracking Analyser (Particle Metrix, Germany) along with the software ZetaView 8.04.02 SP1. Prior to the analysis, the instrument was caliberated using
$100 \mathrm{~nm}$ polystyrene nanostandard particles and cell quality checking was performed before sample reading. The video was captured at a shutter speed of 100 , sensitivity to 85 and frame rate of 30 . Size (diameter in $\mathrm{nm}$ ) and concentration (particles/ml) for each sample was determined by injecting the diluted sample in filterd PBS, with 2 cycles reading at each position. The values obtained from the analyzer were finally calculated by multiplying with the dilution factor.

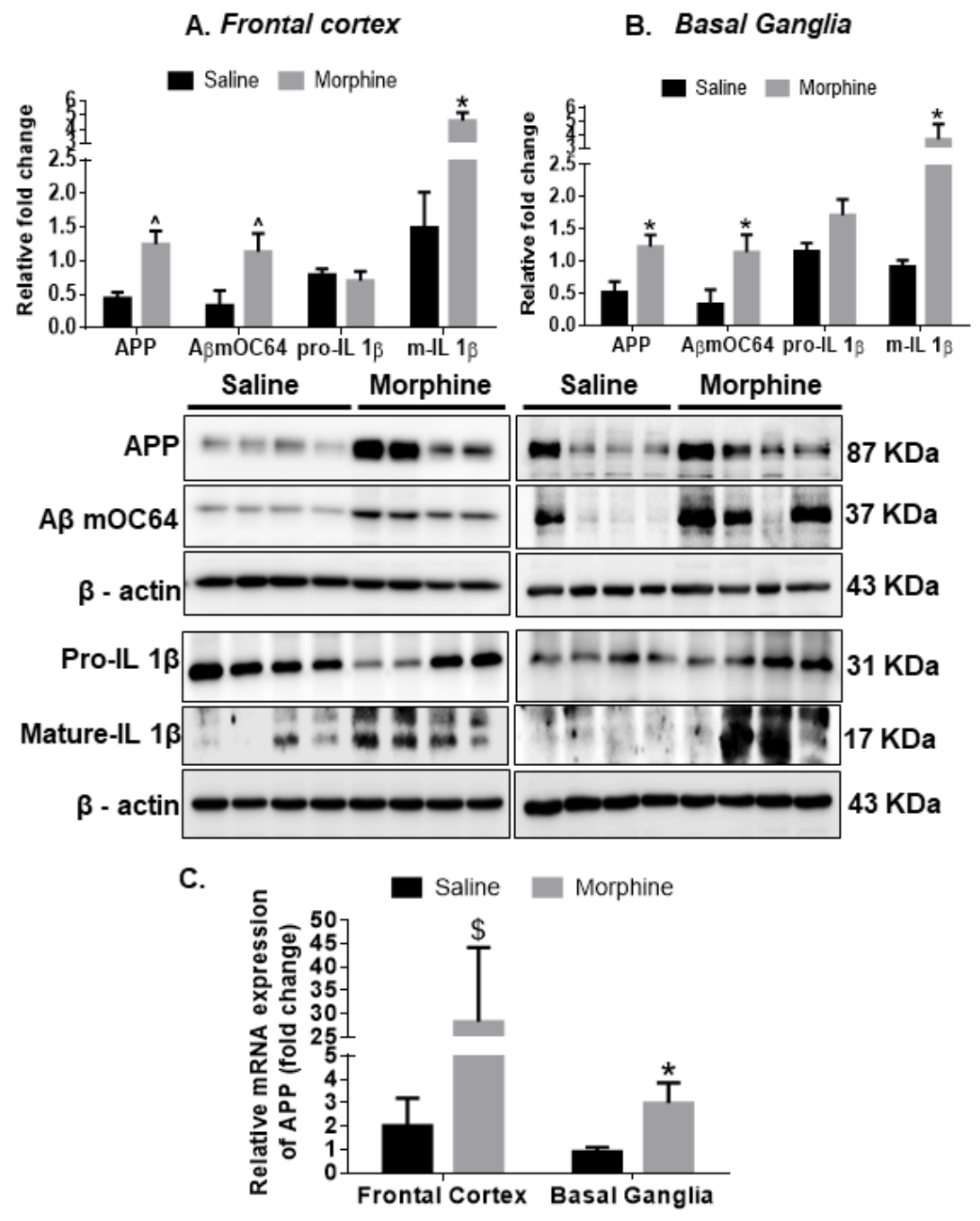

Figure 1. Amyloidopathy in the brain regions of morphine-dependent macaques. Representative western blots showing the expression of APP, A $\beta$ mOC64, pro-IL1- $\beta$ and mature- IL1- $\beta$ in (A) frontal cortex (FC) and (B) basal ganglia (BG) of saline and morphine dependent macaques. (C) qPCR analysis showing expression of APP mRNA in the FC and BG of saline and morphine dependent macaques. $N=4$ macaques per group. Data are presented as mean \pm SEM. Student $t$ test was used to determine the statistical significance between the groups: \$P $<0.01, * \mathrm{P}<0.05$ versus saline. Abbreviations: APP, Amyloid Precursor Protein, A $\beta$, amyloid $\beta$, IL1- $\beta$, interleukin, qPCR, quantitative polymerase chain reaction.

\section{Electron Microscopy}

Negative staining was performed with EV pellets with slight modifications. Briefly, EV pellets $(3 \mathrm{ml})$ were deposited on 200-mesh Formvar-coated copper grids and the membranes were covered for 4-5 minutes for the absorption. The grids were further transferred to uranyl acetate solution for contrast staining. Hereafter, the grids were washed with PBS and excess fluid was blotted with

filter paper and allowed to air-dry at room temperature. Imaging was performed using Hitachi H7500 electron microscope (Hitachi, Tokyo, Japan) at 200kV.

\section{Atomic Force Microscopy}

The 1-(3-aminopropyl) silatrane (APS) was functionalized on freshly cleaved mica surface by depositing $500 \mu \mathrm{M}$ APS solution onto the mica and 
incubating for $30 \mathrm{~min}$ with a wet cap on it as described previously [56]. EV samples $\left(20 \mu 1,8.3 \times 10^{9} \mathrm{EVs} / \mathrm{ml}\right)$ were deposited on APS functionalized mica and incubated for $20 \mathrm{~min}$ at normal room temperature (RT). The sample was then rinsed with PBS and was subjected to imaging using Asylum Research MFP3D instrument equipped with MSNL ' $E$ ' cantilevers with a spring constant of 0.1 $\mathrm{N} / \mathrm{m}$ (Santa Barbara, CA, USA) without allowing the mica surface to get dried.

\section{A. Frontal cortex}

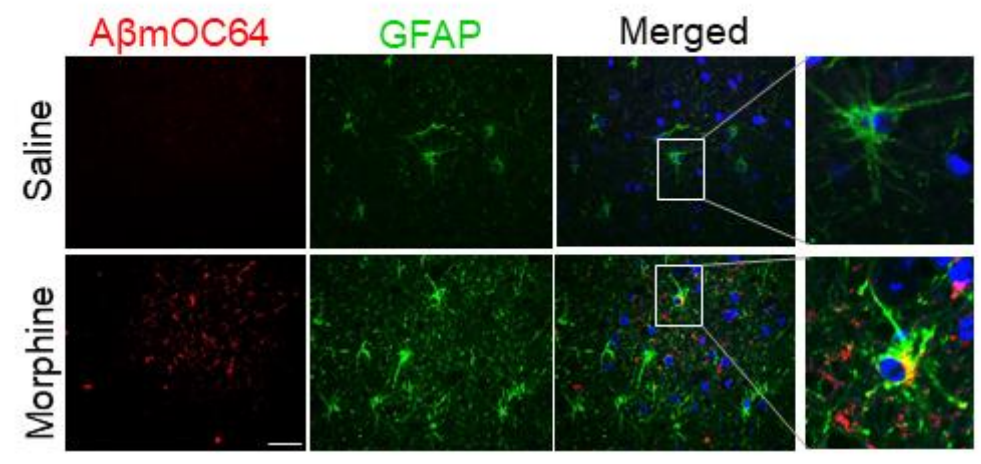

B. Basal Ganglia
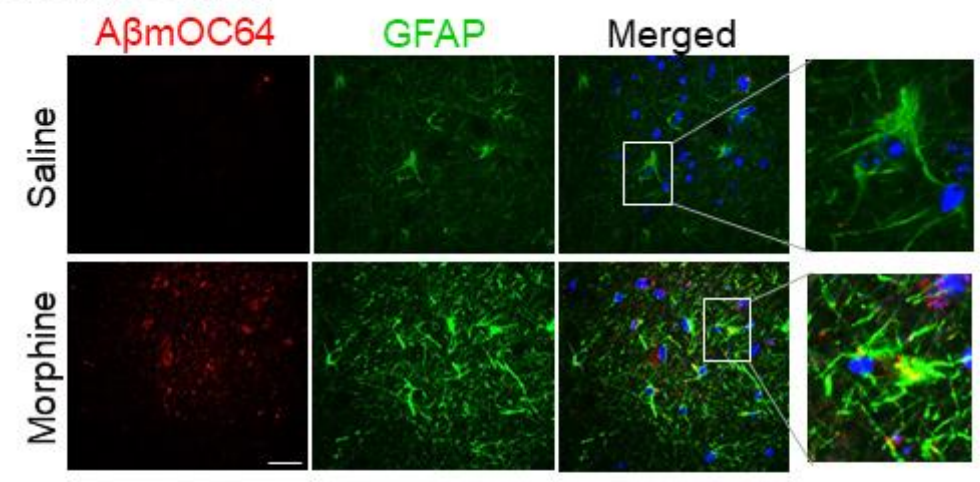

C.
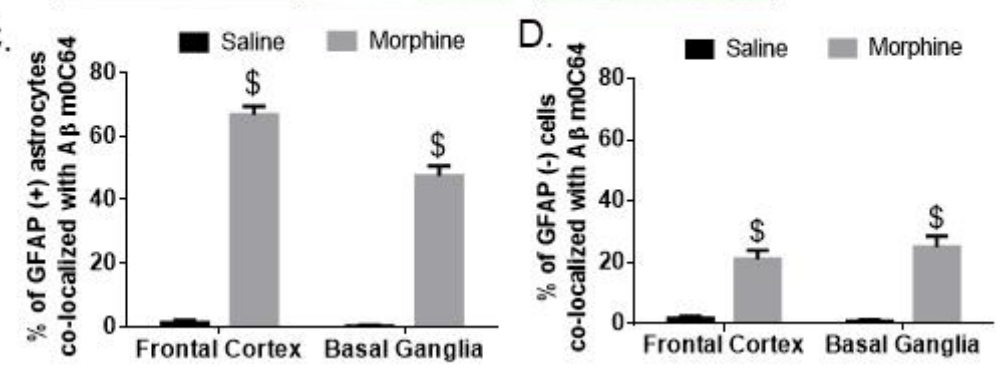

Figure 2. Expression of astrocytic Aß mOC64 in the FC and BG of morphine-dependent macaques. Representative immunohistochemistry photomicrographs showing differential expression of $\mathrm{A} \beta$ mOC6 4 protein in GFAP-positive astrocytes in the FC (A) and BG (B) of morphine-dependent macaques. Scale bar, $20 \mu \mathrm{m}$. Quantitative analysis of percent of GFAP positive (C) and negative astrocytes (D) colocalized with $\mathrm{A} \beta$ mOC64 protein in saline and morphine-dependent macaque. Ten fields from $\mathrm{FC}$ and $\mathrm{BG} /$ macaque were analyzed from $n=4$ macaques per group. Data are presented as mean \pm SEM. Student $t$ test was used to determine the statistical significance: $\$ \mathrm{P}<0.001$ versus saline. Abbreviations: A $\beta$, amyloid beta, GFAP, glial fibrillary acidic protein.

\section{RESULTS}

\section{Morphine-dependent rhesus macaques exhibit brain region-specific amyloidosis}

We first wanted to assess the expression of toxic amyloid proteins in brain homogenates of saline and morphine- dependent rhesus macaques by western blotting. As shown in Fig. 1A-B, there was significant up-regulation of toxic A $\beta$ mOC64 $\wedge^{\wedge}(P<0.01)$, APP $\wedge(P<0.01)$, and pro-inflammatory cytokine IL $1 \beta$ protein $*(P<0.05)$ in the select brain regions- frontal cortex (FC) and basal ganglia (BG) of the morphine administered macaques versus the saline group. Further, qRT-PCR showed that in 
addition to protein levels, mRNA levels of the APP were also significantly upregulated in the frontal cortex $\$(P<$ $0.001)$ and basal ganglia $*(P<0.05)$ in the morphine group compared with the saline group (Fig. 1C).

\section{Immunohistochemistry of A $\beta$ 1-42/ mOC64 expression in brain regions of morphine dependent macaques}

Next, we sought to assess the presence of $A \beta 1-42$ in glial fibrillary acidic protein (GFAP)-positive astrocytes in the brains of macaques. For this, brain sections from saline and morphine dependent macaques were coimmunostained for the presence of A $\beta 1-42 / \mathrm{A} \beta \mathrm{m} 0 \mathrm{C} 64$ and GFAP. As shown in Figs. 2-3, there was increased expression of both varieties of amyloids- A $\beta 1-42$ and $\mathrm{A} \beta \mathrm{m} 0 \mathrm{C} 64$ in the frontal cortex (FC) and basal ganglia
(BG) of morphine dependent animals with a significant $\$(P<0.001) \quad$ colocalization with GFAP-positive astrocytes. Interestingly, we observed that GFAPnegative cells also showed increased expression of $\mathrm{A} \beta 1-$ $42 *(P<0.05)$ as well as A $\beta m 0 \mathrm{C6} 4 \$(P<0.001)$, indicating that cells other than astrocytes could also contribute to the process of amyloidosis. Quantitative analysis demonstrated that the percent of amyloids in GFAP negative cells was between $5 \%$ and $25 \%$ while that for GFAP positive cells was between $40 \%$ and $75 \%$. We also found that several amyloids did not colocalize with the DAPI-nucleus, thereby indicating extracellular deposition of amyloids. Lower magnification images of $\mathrm{A} \beta 1-42$ and $\mathrm{A} \beta \mathrm{m} 0 \mathrm{C} 64$ colocalizing with GFP positive astrocytes in the FC and BG of morphine dependent animals are shown in Supplementary Figs. 1-2.

\section{A. Frontal cortex}

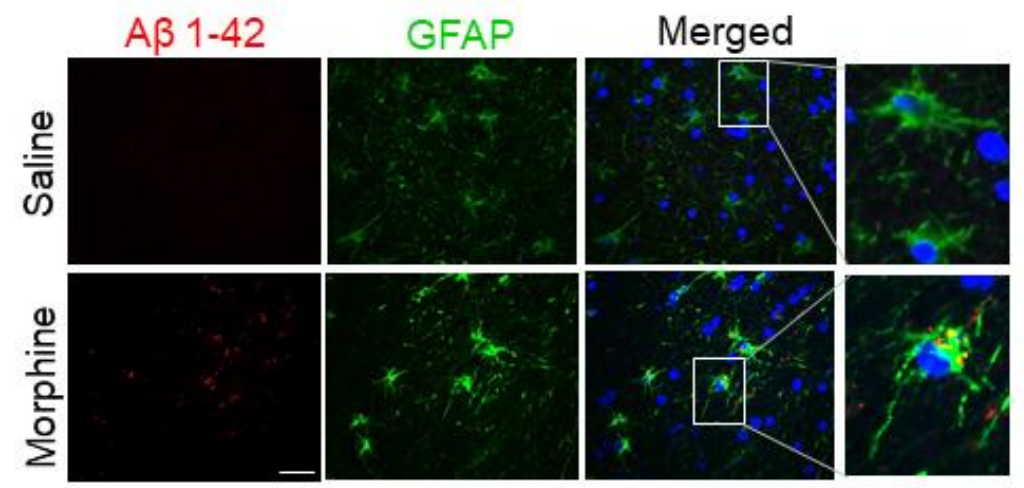

B. Basal Ganglia
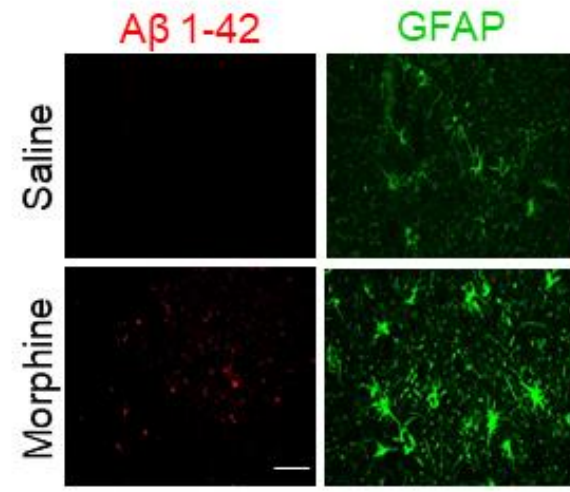

\section{Merged}

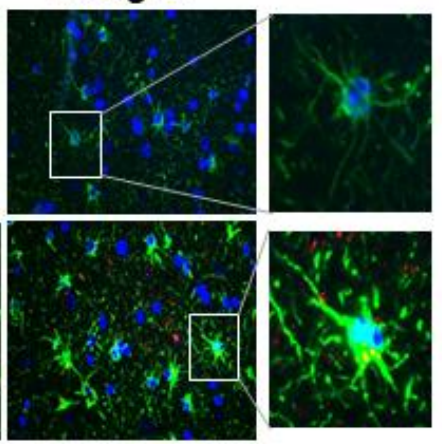

C.

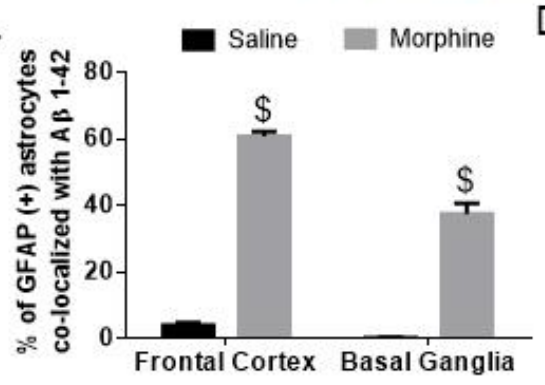

D.

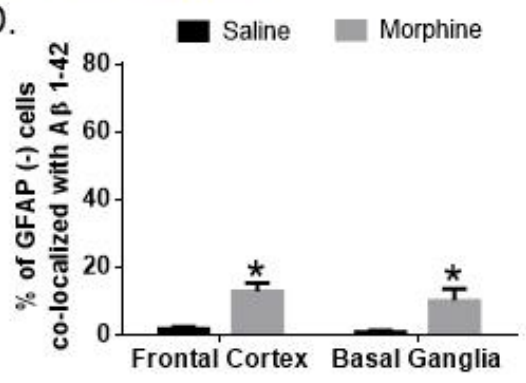

Figure 3. Expression of astrocytic A $\beta$ 142 in the FC and BG of morphinedependent macaques. Representative immunohistochemistry photomicrographs showing differential expression of $\mathrm{A} \beta$ 142 protein in GFAP-positive astrocytes in the FC (A) and BG (B) of morphinedependent macaque. Scale bar, $20 \mu \mathrm{m}$. Quantitative analysis of percent GFAP positive (C) and negative (D) astrocytes colocalized with $A \beta 1-42$ protein in saline and morphine-dependent macaque FCs and BGs. Ten fields from FC and BG/macaque were analyzed from $n=4$ macaques. Data are presented as mean \pm SEM. Student $t$ test was used to determine the statistical significance: $\$ \mathrm{P}<$ $0.001, \quad * \mathrm{P}<\quad 0.05 \quad$ versus saline. Abbreviations: $\mathrm{A} \beta$, amyloid beta, GFAP, glial fibrillary acidic protein. 
A.
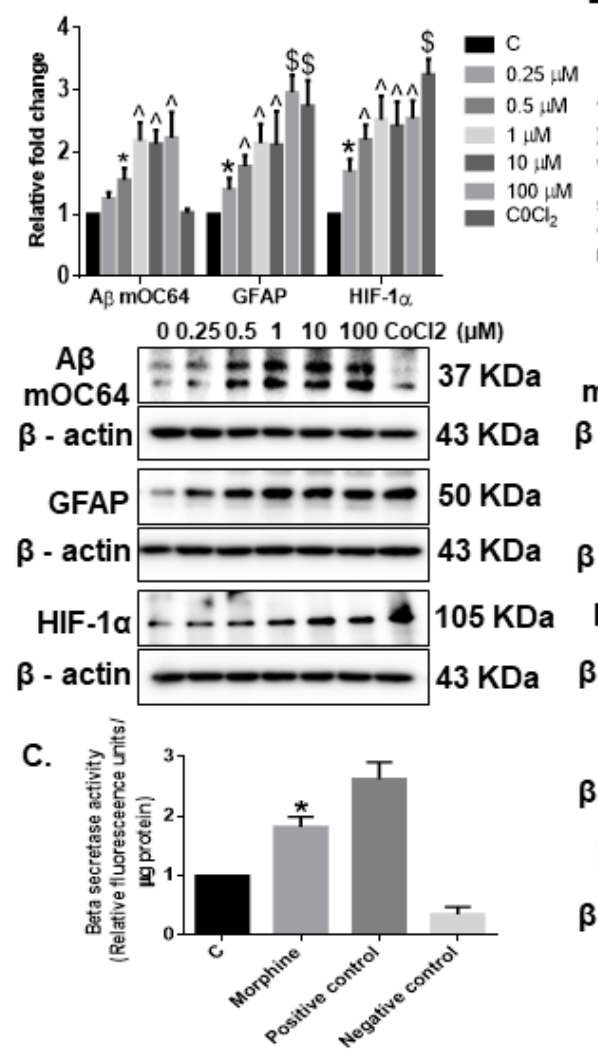

D.

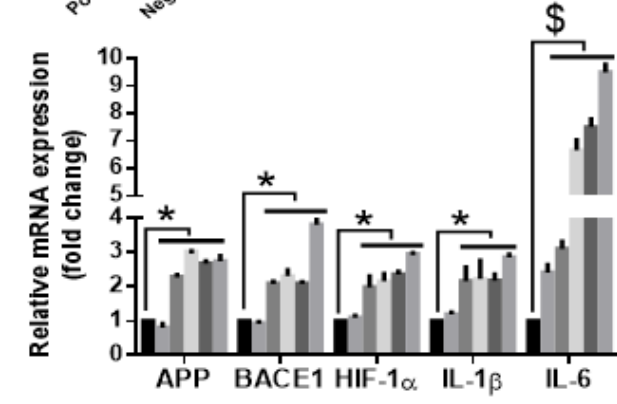

\section{Morphine exposure induces astrocytic amyloidosis}

Based on our ex vivo findings, next we sought to explore the molecular mechanisms associated with morphineinduced astrocytic amyloidosis in vitro. HIF-1 $\alpha$ [57] is a known inducer of BACE1 enzyme, which is crucial for sequential cleavage of $A \beta$ from APP [20-22]. Herein human primary astrocytes (HPAs) were exposed to varying concentrations of morphine $(0,0.25,0.5,1,10$, $100 \mu \mathrm{M}$ ) for 24 hours followed by assessment of cell lysates for the expression of A $\beta$ mOC64, GFAP, HIF- $1 \alpha$. As shown in Fig. 4A, morphine at $0.5 \mu \mathrm{M}$ concentration significantly $\left(* P<0.05,{ }^{\wedge} P<0.01, \$ P<0.001\right)$ increased the expression of conformation-specific $A \beta$ mOC64 and HIF- $1 \alpha\left(\mathrm{CoCl}_{2}\right.$ was used as a positive control for HIF- $1 \alpha$ expression) as well as GFAP in a dose-dependent mannercompared with control cells not exposed to morphine. It must be noted that this concentration $(0.5 \mu \mathrm{M})$ of morphine is in keeping with the physiological levels of morphine found in the postmortem brain tissues of narcotic overdose individuals ( $200 \mathrm{ng} / \mathrm{g}$ of brain tissue) [58]. Since $0.5 \mu \mathrm{M}$ morphine showed consistent and significant up-regulation of all the markers and closely matches with the concentration of morphine in the brains of opiate abusers, this concentration was chosen for future experimentation. Next, we wanted to determine the timedependent $(3,6,12,24,48$ hours) expression of amyloid markers in astrocytes exposed to $0.5 \mu \mathrm{M}$ of morphine. As shown in Fig. 4B, morphine $(0.5 \mu \mathrm{M})$ significantly increased $*(P<0.05)$ the expression levels of $\mathrm{A} \beta$ mOC64, APP $\$(P<0.001)$, HIF-1 $\alpha$, BACE1 and GFAP in a timedependent manner in HPAs, with consistent up-regulation at 24 hours post-morphine exposure compared with control cells. Based on these findings, the optimal dose 
and time for morphine effect was $0.5 \mu \mathrm{M}$ for 24 hours which was the chosen dose and time for all subsequent experiments. Morphine exposure was also found to significantly up-regulate $*(P<0.05)$ BACE1 activity in HPAs (Fig. 4C). Similar to findings in Fig 3A-C, morphine exposure also mediated induction of astrocytic amyloidosis by significantly upregulating $*(P<0.05)$ the mRNA expression of APP, BACE1 and HIF- $1 \alpha$ as well as neuroinflammatory cytokines IL $1 \beta$ and IL $6 \$(P<0.05)$ in HPAs exposed to morphine (Fig. 4D).

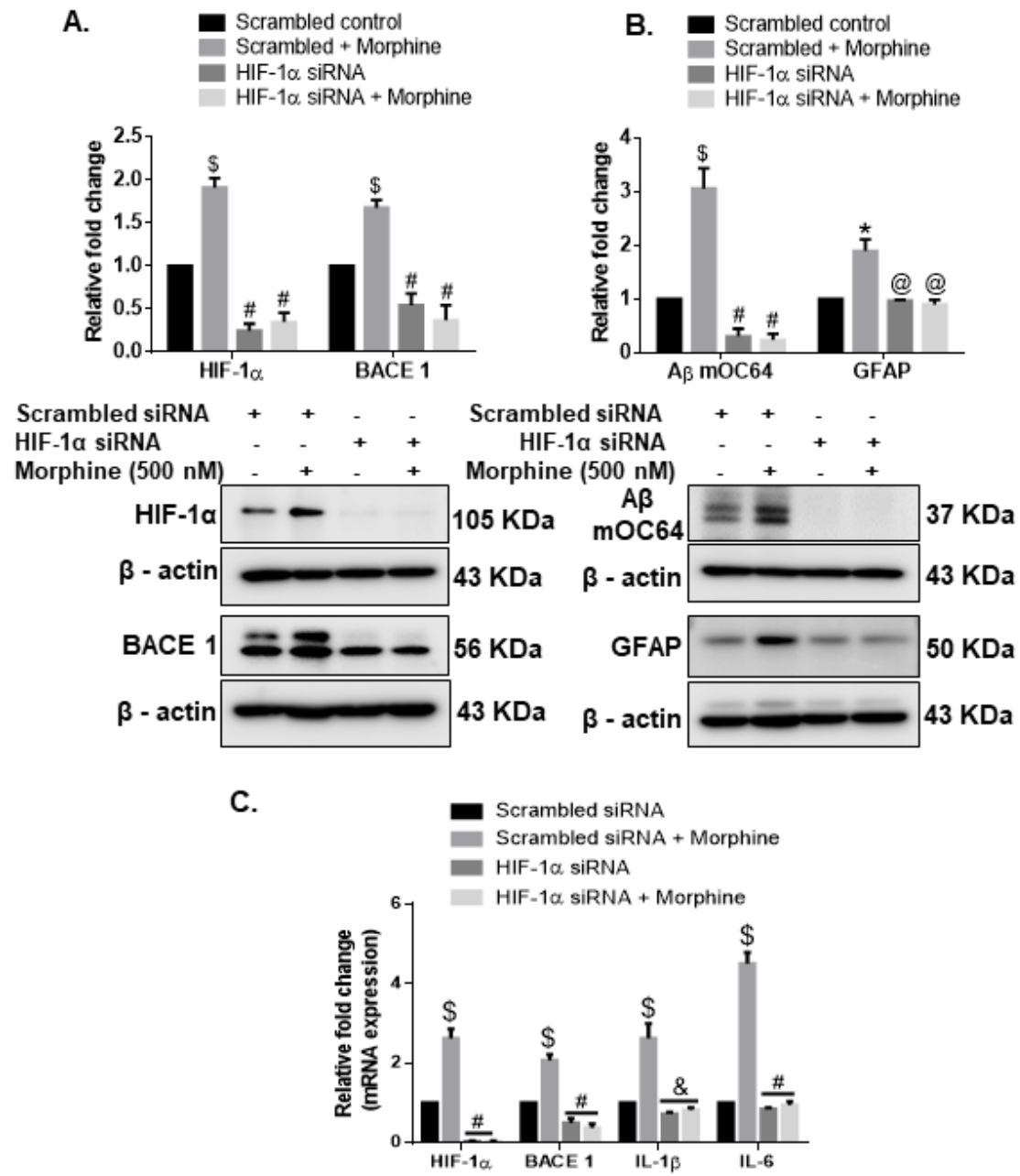

Figure 5. Role of HIF-1 $\alpha$ in morphinemediated astrocytic amyloidosis. Representative western blots showing the expressions of (A) HIF-1 $\alpha$ and BACE1 and (B) A $\beta$ mOC64 and GFAP in HPAs transfected with either HIF-1 $\alpha$ siRNA or scrambled siRNA in the presence of morphine $(500 \mathrm{nM})$. (C) qPCR showing expression of HIF-1 $\alpha$, BACE1, IL1- $\beta$, and IL1-6 RNA in HPAs transfected with either HIF- $1 \alpha$ or scrambled siRNA. Data are presented as mean \pm SEM. One-way ANOVA followed by Bonferroni post hoc test was performed, $\$ \mathrm{P}<0.001, * \mathrm{P}<0.05$ versus control, $\# \mathrm{P}<0.001, \& \mathrm{P}<0.01$ versus scrambled siRNA+Morphine. Abbreviations: $\mathrm{A} \beta$, amyloid beta, HIF- $1 \alpha$, hypoxia-inducible factor $1 \alpha$, HIF- $1 \alpha$, hypoxia-inducible factor $1 \alpha$, HPA, human primary astrocyte, IL, interleukin, qPCR, quantitative polymerase chain reaction, siRNA, small interfering RNA.

\section{HIF-1 1 regulated expression of toxic amyloid forms \& neuroinflammation involves BACE1 in HPAs}

Having determined that morphine can induce the expression of HIF-1 $\alpha$ in HPAs, we next wanted to determine the role of HIF- $1 \alpha$ in inducing the expression of BACE1, using the gene silencing approach. For this HPAs were transfected with either HIF-1 $\alpha$ small interfering RNA (siRNA) or scrambled siRNA followed by exposure of cells to morphine $(0.5 \mu \mathrm{M})$ and assessed for expression of HIF- $1 \alpha$, BACE1, IL $1 \beta$, IL 6 mRNA as well as intracellular expression of A $\beta$ mOC64, BACE1, HIF- $1 \alpha$ and GFAP. Scrambled siRNA-transfected HPAs followed by morphine exposure resulted in significant increases $\$(P<0.001)$ in the mRNA expression of HIF-
$1 \alpha$, BACE1, IL $1 \beta$, IL 6 , as well in the protein levels of HIF-1 $\alpha$, BACE1, A $\beta$ mOC64 and GFAP $*(P<0.05)$, compared with cells not exposed to morphine. HPAs transfected with HIF-1 $\alpha$ siRNA in the presence of morphine exposure demonstrated significant decreases $\#(P<0.001)$ in the expression of HIF- $1 \alpha$, BACE1, IL $1 \beta$ $\&(P<0.01)$, IL 6 RNA as well as decreased expression of HIF-1 $\alpha$, BACE1, A $\beta$ mOC64, and GFAP proteins (Fig. 5A-C) compared with HPAs transfected with scrambled siRNA and exposed to morphine. To further validate these findings, we next silenced the BACE1 gene by transfecting the HPAs with BACE1 siRNA, followed by exposure of cells to morphine. As shown in Fig. 6A-C, in BACE1 siRNA-transfected HPAs in the presence or absence of morphine, demonstrated significantly 
decreased $\#(P<0.001)$ expression of BACE1, IL $1 \beta$, IL 6 RNA and BACE1, A $\beta$ mOC64 and GFAP $@(P<0.05)$ protein levels compared with morphine-exposed HPAs transfected with scrambled siRNA. Expression of HIF1- $\alpha$ in siRNA-transfected HPAs, however, remained unchanged in the presence of morphine. Gene silencing approach underpinned the role of HIF- $1 \alpha$ as a regulator of BACE1 expression in presence of morphine. This was further confirmed by bioinformatic analysis which demonstrated presence of HIF-1 $\alpha$ binding motif on the promoter of BACE1 (Fig. 6D).

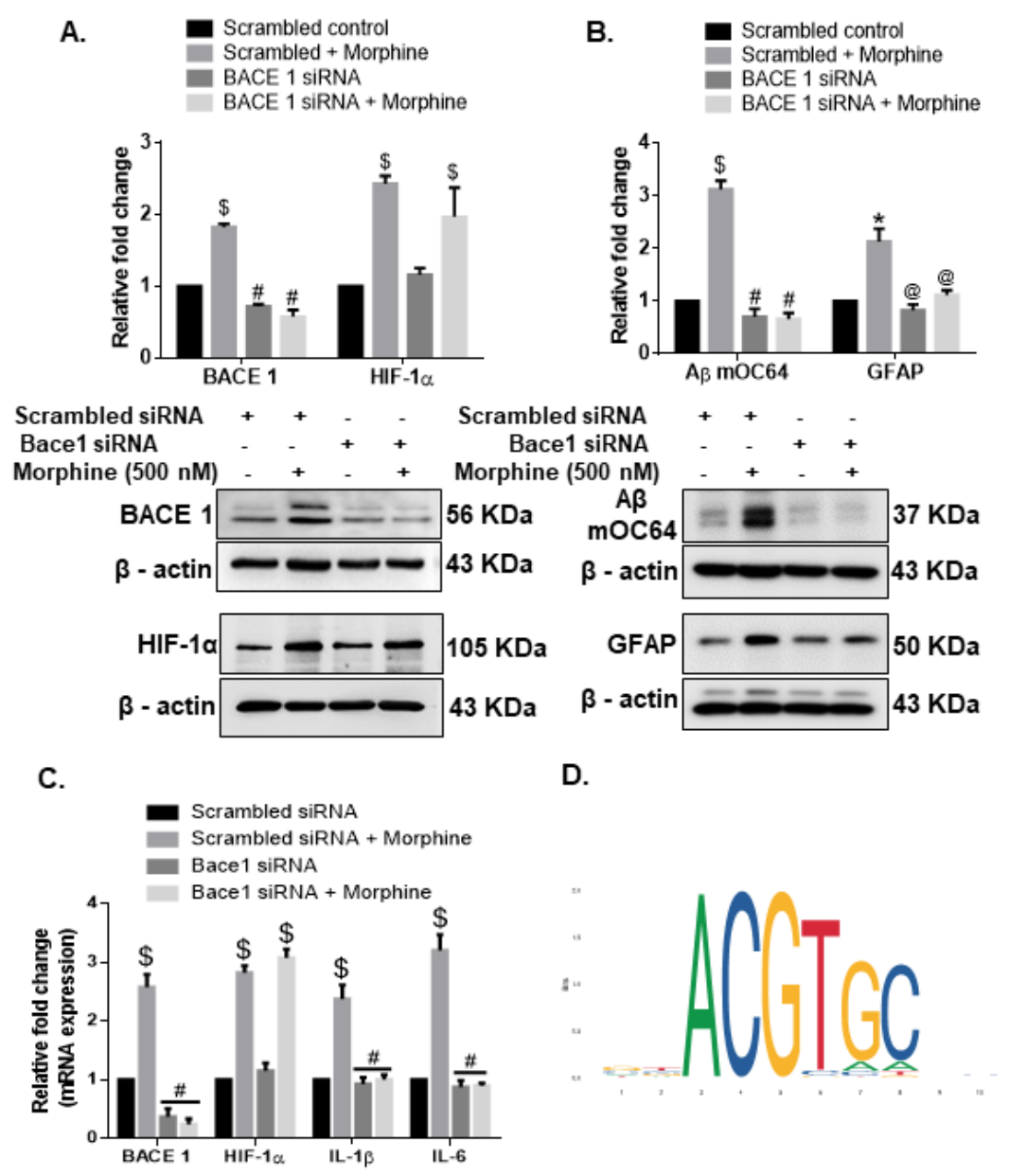

\begin{abstract}
Figure 6. Role of BACE1 in morphinemediated astrocytic amyloidosis. Representative western blots showing the expressions of (A) BACE1 and HIF$1 \alpha$ (B) A $\beta$ mOC64 and GFAP in HPA transfected with either BACE1 siRNA or scrambled siRNA in the presence or absence of morphine (500 nM). (C) qPCR showing expression of BACE1, HIF- $1 \alpha$, IL1- $\beta$, and IL1-6 mRNA in HPAs transfected with either BACE1 or scrambled siRNA. (D) HIF-1 $\alpha$ binding motif on the BACE1 promoter. Data are presented as mean \pm SEM. One-way ANOVA followed by Bonferroni post hoc test was performed, $\$ \mathrm{P}<0.001, * \mathrm{P}<$ 0.05 versus control, \#P< 0.001, @ $\mathrm{P}<$ 0.05 versus scrambled siRNA+ Morphine. Abbreviations: $A \beta$, amyloid beta, HIF-1 $\alpha$, hypoxia-inducible factor $1 \alpha$, HPA, human primary astrocyte, IL, interleukin, siRNA, small interfering RNA.
\end{abstract}

\section{Increased expression of HIF-1 $\alpha$ and BACE1 in the FC and BG of morphine-dependent rhesus macaques}

Having determined the role of HIF-1 $\alpha$ in mediating the expression of BACE1 in the presence of morphine, we next sought to assess the expression of both HIF- $1 \alpha$ and BACE1 by western blotting in select brain regions (FC and $\mathrm{BG}$ ) of morphine-dependent and saline control macaques. As shown in Fig. 7A-B, there was significant up-regulation $*(P<0.05)$ of HIF-1 $\alpha$ and BACE1 proteins in both the $\mathrm{FC}$ and $\mathrm{BG}$ of morphine administered macaques compared with the saline controls. Furthermore, there was a significant upregulation in both HIF- $1 \alpha *(P<0.05)$ and BACE1 $*(P<0.05)$ mRNAs in the frontal cortex, HIF- $1 \alpha^{\wedge}(P<0.01)$ and BACE1 $\$(P<$
0.001) mRNAs in the basal ganglia of the morphinedependent macaques compared with saline (Fig. 7C-D).

\section{Morphine administration induced the release of ADEVs carrying the amyloid cargoes in HPAs}

Herein we sought to determine whether ADEVs released from morphine-stimulated HPAs could also contain the amyloid cargoes. Astrocytes have been shown to produce considerable amounts of EVs [59] and were thus chosen for this study. For this we first isolated and characterized ADEVs from conditioned media of HPAs, using a differential ultracentrifugation procedure (Fig. 8A) [60, 61]. Purified EVs were quantified in Nanoparticle Tracking Analysis (NTA). As shown, the numbers of both $100,000 \times$ g-exosomes (Control-ADEV 0.5975 $\times 10^{9} \pm$ 
$0.1855 \times 10^{9}$ and morphine exposed ADEV $3.850 \times 10^{9} \pm$ $1.060 \times 10^{9}$ ) and $167,000 \times$ g-exomeres (Control-ADEV $0.3725^{*} 10^{9} \pm 0.2456 * 10^{9}$ and morphine exposed ADEV $\left.4.475 \times 10^{9} \pm 1.580 \times 10^{9}\right)$ were significantly $\$(\mathrm{p}<0.001)$ increased in supernatants obtained from cells exposed to morphine $(0.5 \mu \mathrm{M}, 24 \mathrm{~h})$ compared with the controls (Fig. $8 \mathrm{~B})$. However, as expected, morphine treatment did not affect the release of $2000 \times \mathrm{g}$ and $10,000 \mathrm{x} \mathrm{g}$ non-EV particles compared to control group not treated with morphine. Immunoblotting of the EV lysates revealed the presence of exosomal markers - TSG101, CD63 and Alix for the $100,000 \times \mathrm{g}$ vesicles, and exomere markers CD63, Flotillin and Ago-2 for the 167,000 xg exomeres (Fig. 8C). Additionally, immunoblotting using calnexin was also assessed to rule out contamination of ADEVs with the cell debris. Next, the EVs were further characterized by transmission electron microscopy (TEM) and atomic force microscopy (AFM) Fig. 8D-F and as presented in Table 1demonstrated that EVs had an average diameter of $103 \mathrm{~nm}$ for exosomes, and $48 \mathrm{~nm}$ for exomeres (Table 1).

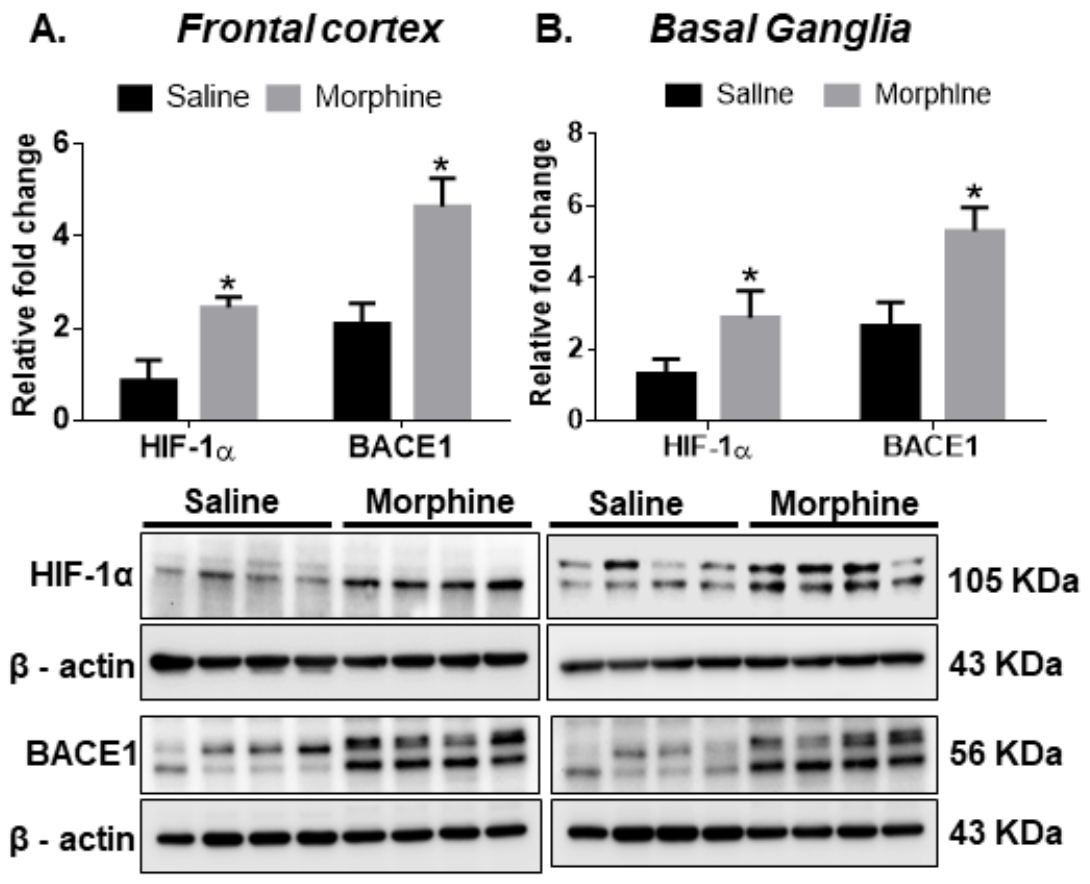

Figure 7. Expression of HIF-1 $\alpha$ and BACE1 in the brains of morphinedependent macaques. Representative western blots showing the expression of HIF- $1 \alpha$ and BACE1 in the FC (A) and BG (B) of saline or morphine-dependent macaques. (C) qPCR demonstrating the expression of HIF- $1 \alpha$ and BACE1 in the FC (C) and BG (D) of saline or morphinedependent macaques. $n=4$ macaques per group. Data are presented as mean \pm SEM. Student $t$ test was used to determine the statistical significance between multiple groups: $\$ \mathrm{P}<0.001,{ }^{\wedge} \mathrm{P}<0.01, * \mathrm{P}<$ 0.05 versus saline. Abbreviations: HIF-1 $\alpha$, hypoxia-inducible factor $1 \alpha$, qPCR, quantitative polymerase chain reaction.

\section{Frontal cortex}

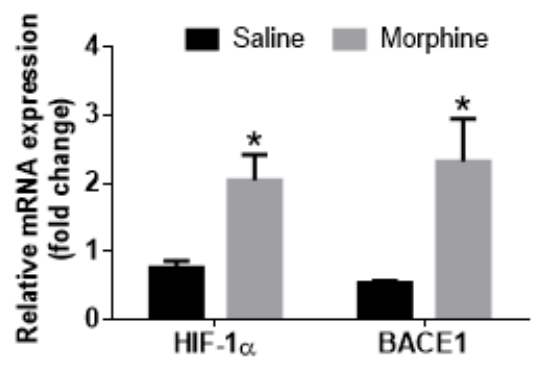

\section{Basal Ganglia}

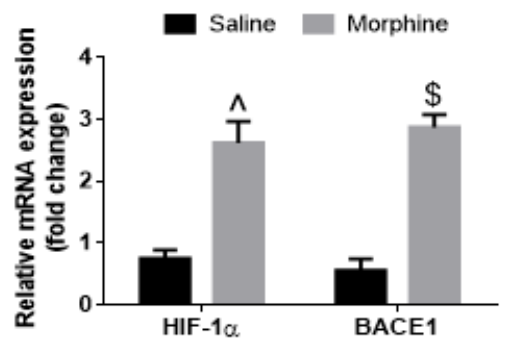

Based on our findings that morphine-mediated induction of HIF-1 $\alpha$ regulated downstream amyloid production in HPAs, we next sought to assess the role of HIF- $1 \alpha$ in morphine-mediated release of ADEVs. For this we used genetic silencing approach to abrogate the expression of HIF-1 $\alpha$ in HPAs followed by assessment of ADEV numbers released by the HPAs. As shown in Fig. $9 A$, there was a significant decrease $\#(p<0.001)$ in the numbers of ADEVs isolated from HIF- $1 \alpha$ silenced HPAs compared with HPAs transfected with scrambled siRNA, in the presence of morphine. Additionally, morphine $(0.5$ $\mu \mathrm{M}, 24 \mathrm{~h}$ ) exposure also increased the packaging of various toxic forms of amyloid cargoes in the ADEVs $(167,000 \mathrm{xg})$ (Fig. 9B). There were no traces of amyloids in ADEVs isolated from HIF-1 $\alpha$ silenced cells. 


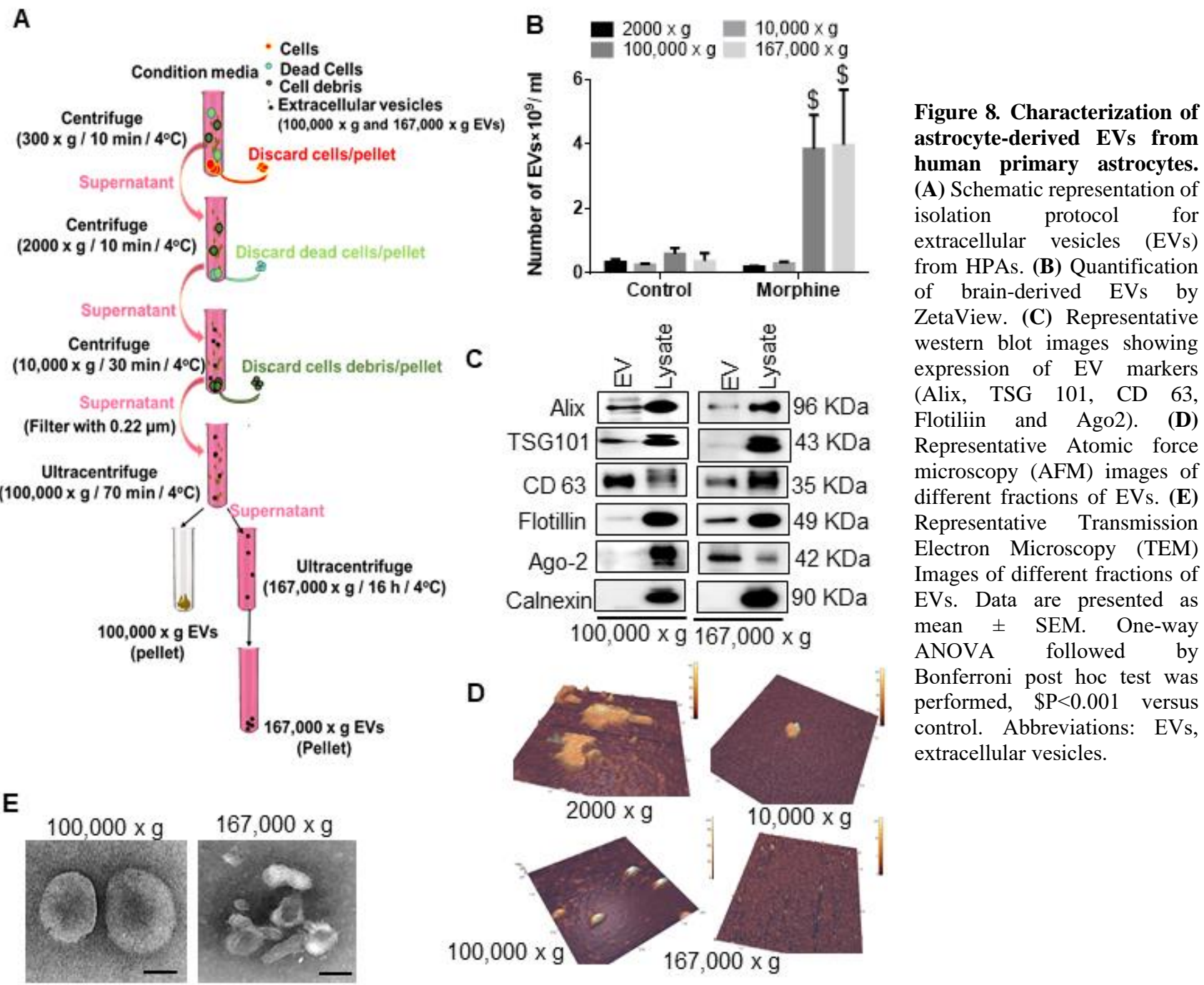

Table 1. Quantitative analysis (width, height, volume) of different fractions of EVs by AFM.

\begin{tabular}{|l|l|l|l|l|}
\hline & $2,000 \times \mathrm{g}$ & $10,000 \times \mathrm{g}$ & $100,000 \times \mathrm{g}$ & $167,000 \times \mathrm{g}$ \\
\hline Width $(\mathrm{nm})$ & $148.1 \pm 85$ & $125.1 \pm 47$ & $103 \pm 53$ & $48 \pm 6$ \\
\hline Height $(\mathrm{nm})$ & $25.8 \pm 12.3$ & $18.9 \pm 15$ & $11.9 \pm 10$ & $10 \pm 3.7$ \\
\hline Volume $\left(\mathrm{nm}^{3}\right)$ & $149731 \pm 116000$ & $75726 \pm 83213$ & $45418 \pm 460000$ & $9324 \pm 3790$ \\
\hline
\end{tabular}

\section{Characterization of brain-derived EVs from saline- and morphine-dependent macaques}

Herein we sought to assess whether brain derived EVs (BEVs) from morphine-dependent macaques also contained amyloid and neuroinflammatory cargoes. BEVs were isolated from the brains of macaques by enzymatic digestion, differential centrifugation, and purification using Optiprep density gradient centrifugation (schematic shown in Fig. 10A). We employed student $t$ - test to determine the statistical significance between saline- and morphine- dependent macaques. Fig. 10B demonstrates the total number of EVs particles present in $\mathrm{mg}$ of the brain tissue in different EV fractions by NTA. Additionally, Fig. 10C demonstrates the size distribution of the EVs in different fractions from per $\mathrm{mg}$ of the brain tissue by NTA. Originally, the count of EVs were determined as present in per $\mathrm{ml}$, and then converted to per $\mathrm{mg}$ of tissue. Size distribution assessed by NTA revealed EVs distribution in the 50-200 $\mathrm{nm}$ range, with maximum 
number of EVs between 100-135 $\mathrm{nm}$ size range. Interestingly, there was no change in numbers or size distribution of BEVs between the saline or morphine groups (Fig. 10B-C). More than $75 \%$ of BEVs ranging in size from 50-200 nm was present in fractions F3-6 (Fig. 10C-D). Western blotting of the BEVs demonstrated the presence of exosome-enriched proteins CD63 and CD9 specifically in F4-6 fractions and were negative for endoplasmic reticulum protein calnexin. Brain-lysate was used as a positive control for calnexin (Fig. 10D). The structure and diameter of the EVs was further confirmed by TEM analysis (Fig. 10E) and the same EV particles with TEM analysis quantification has been represented in
Supplementary Fig. 3A. Loading equal numbers of $\mathrm{BEVs} /$ protein concentration for western blotting from both saline and morphine-dependent animals, demonstrated a significant increase in N-terminally cleaved APP $\wedge(p<0.01), A \beta m O C 64$ (trend of increase) as well as pro- and mature- IL- $1 \beta *(p<0.05)$ in the BEVs from morphine-dependent macaques compared with saline group of macaques (Fig. 10F-G). Non-EV fractions from either of the macaque groups failed to exhibit the presence of the amyloid cargoes (Supplementary Fig. 3B). No significant change in protein concentration was observed between saline- and morphine- BEVs (Supplementary Fig. 3C).

\section{B.}

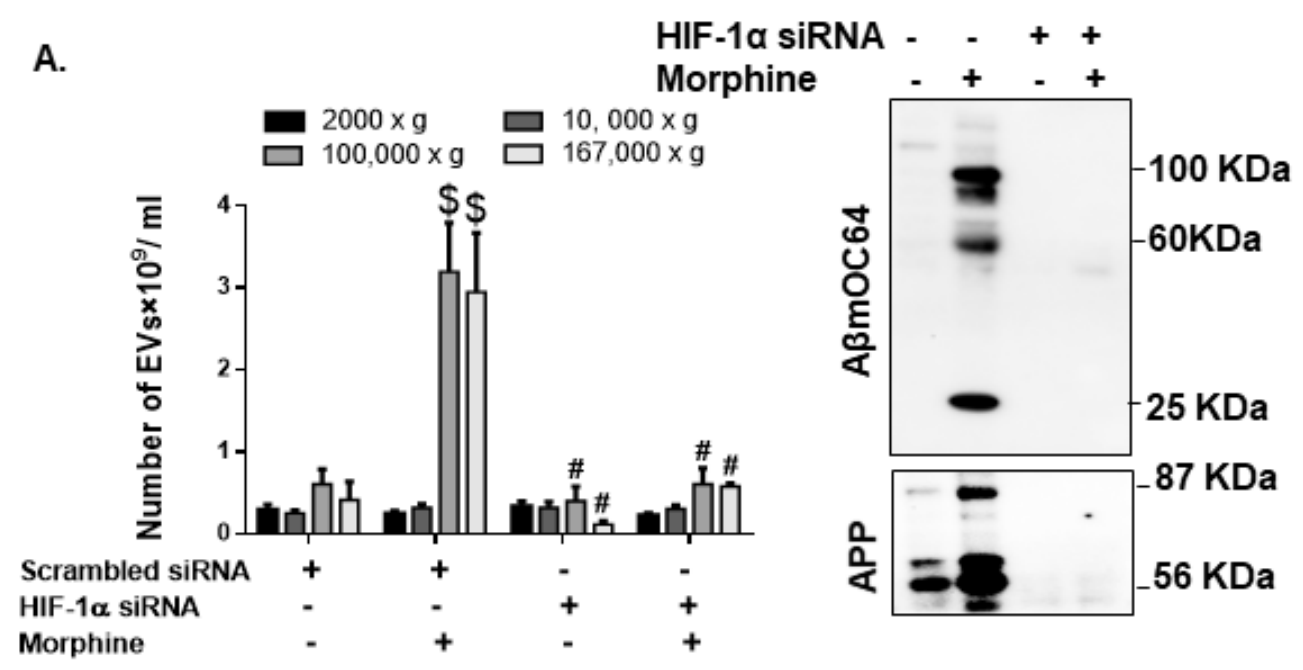

Figure 9. Role of HIF-1 $\alpha$ in morphine mediated EV release and cargoes. (A) Quantitative analysis of EV numbers isolated from condition media of HPAs transfected with either scrambled siRNA or HIF$1 \alpha$ siRNA followed by exposure to morphine. (B) Representative western blot images showing protein levels of A $\beta$ mOC64 and APP in HPAs transfected with either scrambled or HIF- $1 \alpha$ siRNA followed by exposure to morphine. Data are presented as mean \pm SEM. One-way ANOVA followed by Bonferroni post hoc test was performed, $\$ \mathrm{P}<0.001$ versus control, $\# \mathrm{P}<0.001$ versus scrambled siRNA+Morphine. $* \mathrm{P}<0.05$ versus control. Abbreviations: APP- amyloid precursor protein, A $\beta$ - amyloid beta, HIF- $1 \alpha-$ hypoxia-inducible factor, siRNA- small interfering RNA.

\section{DISCUSSION}

Chronic opiate use has been reported to cause multitude of cognitive deficits including impairment of attention, working memory, episodic memory $[15,16]$ as well as prospective memory [12]. On an average $\sim 20-44 \%$ of the cancer patients taking morphine for pain control go on to develop cognitive decline [14]. Recent reports have demonstrated the presence of hyperphosphorylated Tau in the brains of chronic opiate users [24] and amyloid deposition was also observed in morphine-dependent rats [62]. Toxic amyloids have been reported to contribute to neurodegeneration and cognitive deficits observed in $\mathrm{AD}$ [63]. The present study for the first time demonstrates the role of astrocytes in regulating morphine-mediated amyloidosis with the involvement of HIF- $1 \alpha$-BACE1 axis, ultimately resulting in neuroinflammation. These findings were also validated in morphine-dependent macaques. Furthermore, we also report dissemination of morphine induced neuropathogenesis by ADEVs carrying the amyloid cargoes.

Astrocytes are an abundant cell type in the CNS, which are involved in several critical physiological functions in the brain such as neurogenesis, synaptogenesis, controlling the blood-brain barrier permeability and maintaining extracellular homeostasis [64-67]. Recent findings have demonstrated the role of astrocytes in morphine-mediated neuroinflammation via 
dysregulated autophagy pathway [50]. The present study demonstrates a unique role of astrocytes in morphinemediated amyloid pathology which leads to neuroinflammation. We first examined astrocytic amyloidosis in the archival tissue sections of morphine dependent rhesus macaques ex vivo. In this study we have shown amyloid pathogenesis in both the frontal cortex (FC) and basal ganglia (BG), since these regions are involved in regulation of learning and memory $[68,69]$ as well as opiate addiction [70-72]. We observed increased amyloidosis as evidenced by immunostaining and western blotting for amyloids, specifically in the FC and BG of morphine-dependent compared with the saline administered macaques. These findings were also validated by increased mRNA expression of APP, BACE1 and HIF-1 $\alpha$ RNA in the brains of morphine dependent macaques. Furthermore, we also validated our findings by immunostaining, wherein we demonstrated increased co-localization of GFAP positive astrocytes with the two varieties of neurotoxic amyloids- $\mathrm{A} \beta \mathrm{m} 0 \mathrm{C} 64$ $\& A \beta 1-42$, thus underscoring the role of astrocytes in morphine-induced amyloidosis. Quantitative analysis further confirmed that the percentage of GFAP+ astrocytes co-localizing with amyloids in these brain regions was significantly higher than the non-astrocytic cells, again validating the vital role of astrocytes in morphine-induced amyloid pathology, which, in turn, could be a key factor underlying cognitive deficits observed in opiate users. Interestingly, we also found higher expression of neuroinflammatory cytokines in the FC and BG of morphine dependent macaques [50]. Upregulation in the protein levels of IL-1 $\beta$ (in the FC and BG of morphine-dependent macaques) was also observed in the present study, thus indicating a positive correlation between morphine-induced amyloidosis and neuroinflammation.
A.
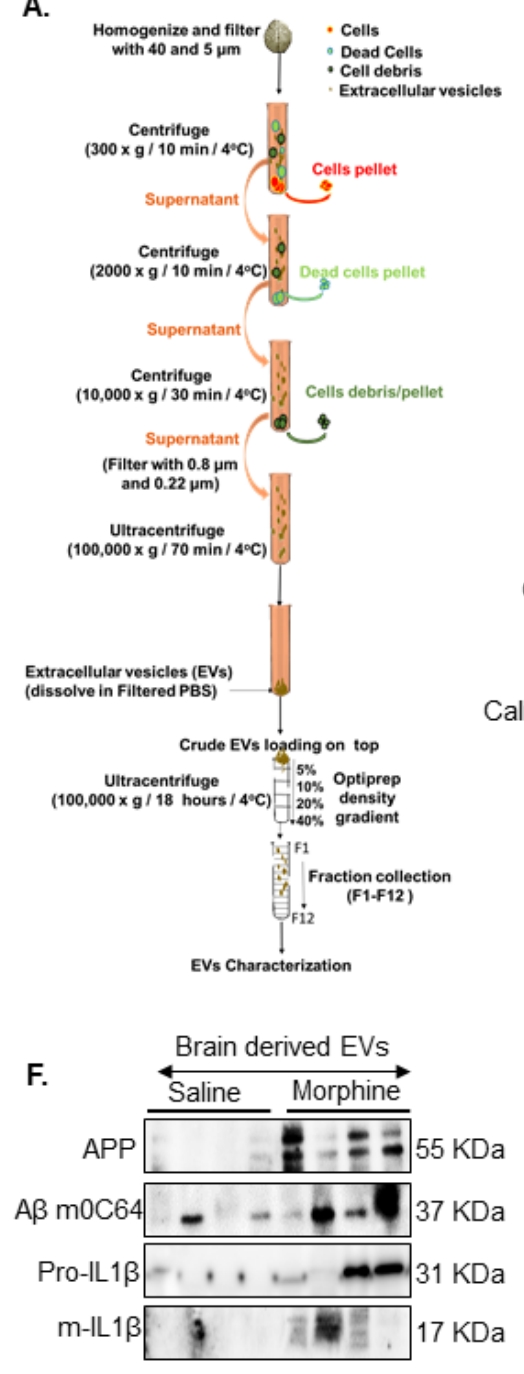

B. $2.5 \times 10^{8}$

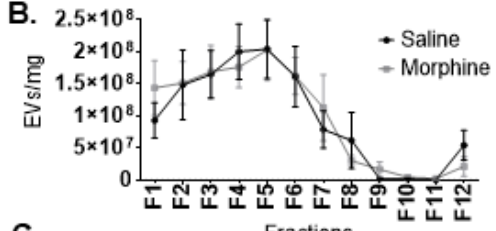

C.

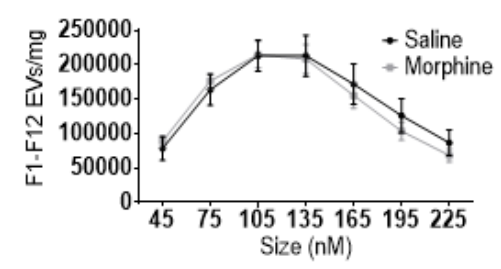

D.

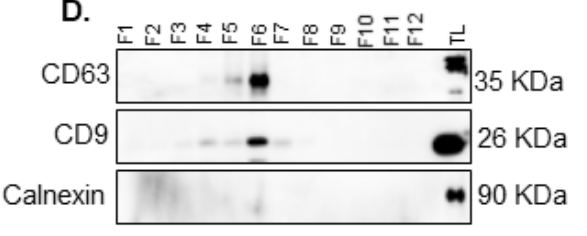

E.

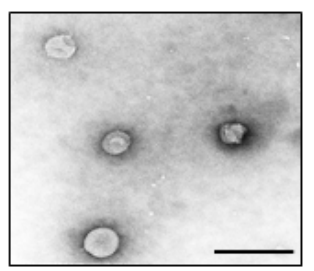

G.

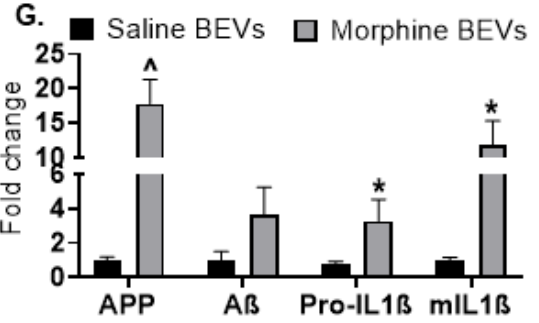

Figure 10. Characterization of EVs from macaque brain. (A) Schematic representation of isolation protocol for EVs from brain of macaques. (B) Quantification of EVs per mg brain tissue from different fractions (F1-F12) assessed by ZetaView. (C) Size distribution of EVs for the size range 45-225 nm (F1-F12) assessed by ZetaView. (D) Representative western blot images showing EV specific markers (CD63, CD9) in different fractions (F1-F12). (E) Topographic profiling of F4-F7 EVs using transmission electron microscopy (TM) under tapping mode revealed a heterogeneous population of spherical particles. (F, G) Representative western blot images and quantification (normalization with proteins in the EVs) for APP, A $\beta$ moC64, Pro-IL1 $\beta$, m-IL$1 \beta$ in brain derived EVs (F4-7) from saline or morphine-dependent macaques. $* \mathrm{P}<0.05,{ }^{\wedge} \mathrm{P}<$ 0.01 versus saline. Abbreviations: $A \beta$ - amyloid beta, EVs- extracellular vesicles, ILinterleukin. 


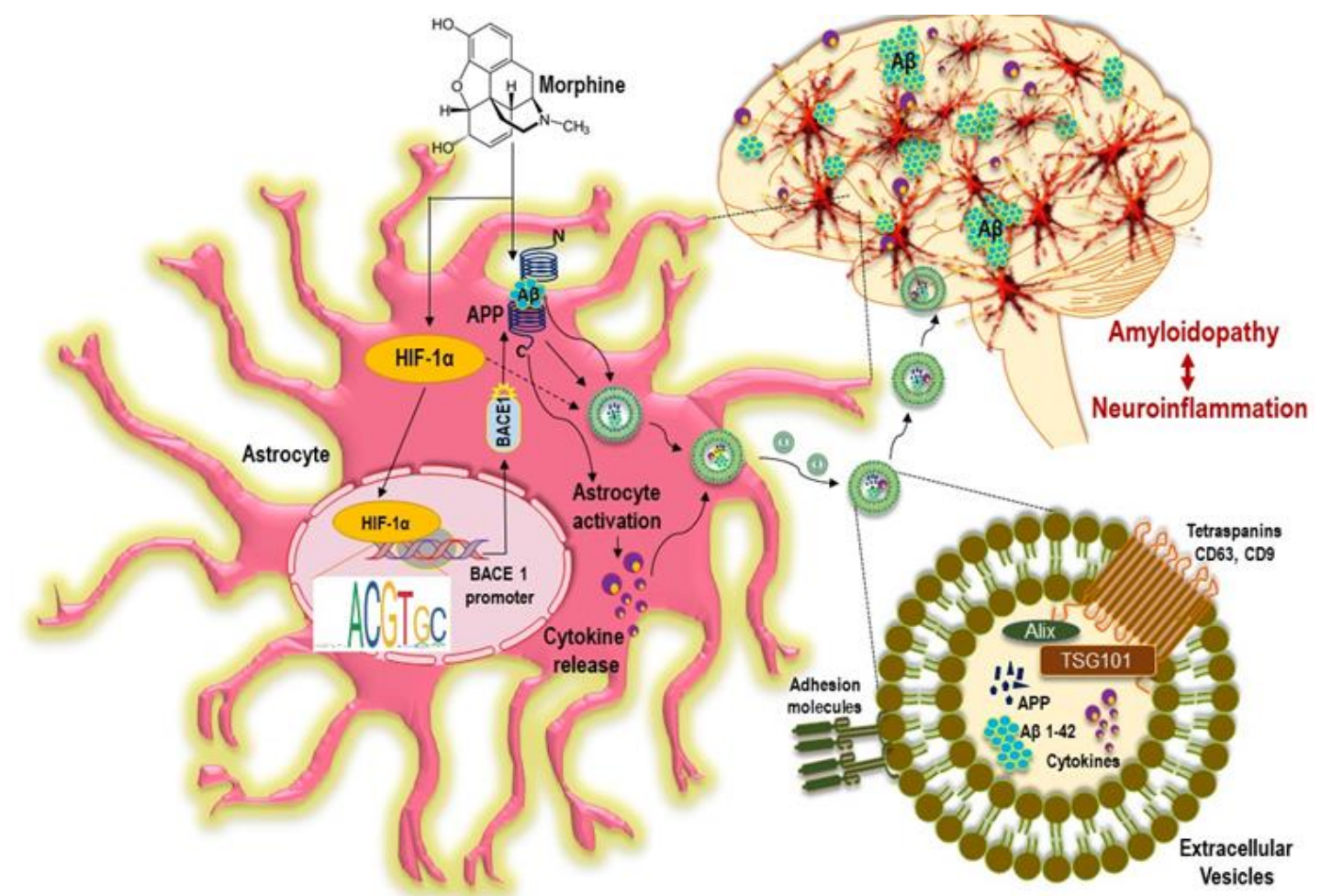

Figure 11. Schematic representation on the role of morphine in extracellular vesicles mediated amyloidopathy and neuroinflammation: Exposure of HPAs to morphine increased the expression and accumulation of HIF-1 $\alpha$ in the cytoplasm. Nuclear translocation of HIF-1 $\alpha$, resulted in the binding of the former on the promoter of BACE1 gene, thus regulating the transcription of the BACE1 gene, which further cleaved the APP to release the A $\beta$. These neurotoxic $\mathrm{A} \beta$, cause astrocyte activation and pro inflammatory milieu in the HPA. Additionally, morphine could induce the release of EVs via HIF- $1 \alpha$ mediated pathway in HPAs. The various forms of amyloids were sorted into the EVs and are transported to different regions of brain resulting in widespread seeding of this amyloids. Additionally, amyloid induced cytokines were also released in the EVs leading to neuroinflammation. Accumulation of toxic $A \beta$ in the brain regions further facilitate neuroinflammation and neurodegeneration, thus contributing to cognitive impairments in opiate users. APP: amyloid precursor protein; A $\beta$ : amyloid beta; BACE1, $\beta$-site cleaving enzyme; HPA, human primary astrocyte, ADEV: astrocyte derived extracellular vesicles.

Our ex vivo findings were further validated in vitro using HPAs. Using these cells, we unraveled the molecular mechanism(s) underlying morphine-mediated astrocytic amyloidosis. In line with our in vivo findings, we found that exposure of HPAs to morphine, resulted in a dose- and time- dependent upregulation of the toxic amyloid forms, thereby underscoring the role of astrocytes in the process of amyloidosis. Additionally, we also observed an increase in BACE 1 activity in morphine exposed HPAs. Our results also showed that morphine increased the expression of APP, BACE1 translationally, while also inducing transcriptional activation of these genes.

Several studies have shown the involvement of amyloidopathy in ischemic brain damage [73, 74]. Furthermore, the involvement of HIF- $1 \alpha$ has also been well-documented in amyloidosis associated with AD [57, 75]. Additionally, in our recent report we have demonstrated the role of HIF- $1 \alpha$, as a master regulator in the process of astrocytic amyloidosis following HIV-Tat exposure [26]. In keeping with our published findings with HIV Tat [26], morphine also induced amyloidosis and upregulated the expression of HIF-1 $\alpha$ in HPAs both transcriptionally as well as translationally. HIF- $1 \alpha$ has been reported to regulate BACE1 expression [26]. It must be noted that while in the non-CNS cells such as Lewis lung carcinoma cells [76] and cardiac myocytes [77] morphine reduces the expression of HIF- $1 \alpha$, however, in both morphine exposed astrocytes as well as in the brains of morphine dependent macaques there was a significant increase in the expression of HIF-1 $\alpha$. Our findings were also validated by bioinformatic analysis which showed HIF- $1 \alpha$ binding motif on the promoter of BACE1, thus adding validation that HIF- $1 \alpha$ can regulate the expression of BACE 1. Using gene silencing approaches we further confirmed HIF- $1 \alpha$ as a master regulator and an upstream mediator that regulated the expression of BACE1 and generation of $A \beta$. It was also found that in addition to regulating amyloid production, the master regulator HIF$1 \alpha$, also regulated astrocyte activation and ensuing 
neuroinflammation. One probable explanation for this could be the intracellular levels of amyloid that could upregulate neuroinflammation [78] or that proinflammatory cytokines could regulate the accumulation of the toxic amyloid forms [78-80].

Another finding of our study was the presence of amyloids in the morphine stimulated ADEVs. EVs are nanoscale double membrane bound vesicles that are released by diverse cell types in the microenvironment [81]. It has been well recognized that astrocytes can communicate via direct cell-cell interactions and gap junctions; however, in recent times, it is being increasingly appreciated that astrocytes can also communicate with other cells within the CNS, through the release as well as uptake of EVs by the recipient cells [82]. These EVs contain surface markers, as well as a biologically active cargo of metabolites, proteins, nucleic acids, and other molecules specific to their tissue (and cell) of origin, reflecting the physiological state of the tissue or the cells [83]. Several studies have shown that these EVs, particularly brain-secreted EVs (BEVs) can carry amyloids, specially in $\mathrm{AD}[84,85]$ and can serve as biomarkers of disease progression. In the present study, for the first time we demonstrate that BEVs from morphine dependent macaques contain varieties of posttranslationally modified A $\beta \mathrm{m} 0 \mathrm{C} 64$ (of different molecular weight) as well as APP, full length and the Nterminally cleaved APP ( $55 \mathrm{KDa})$, both of which are considered to be highly neurotoxic [86]. Additionally, we have found that the cytokines (IL-1 $\beta$ ) which were upregulated in the brains of morphine dependent macaques were also released in the BEVs. Increased numbers of EVs carrying toxic amyloids could likely play a key role in seeding in the different brain regions, thereby leading to progression of disease pathogenesis as well as neurodegeneration and neuroinflammation. Additionally, the cytokines released via the BEVs could also add to the neuroinflammatory burden in the brain. As discussed earlier, several studies have reported a close link between amyloids and neuroinflammation [78-80], such that both the released amyloids and the neuroinflammatory factors can lead to progressive neurodegeneration and neuroinflammation via an inter-related cascade. It was intriguing to note that although the BEVs from saline and morphine dependent macaques did not alter in numbers, size distribution and protein content, there was increased amyloid and neuroinflammatory cargo in morphine BEVs compared to the saline group BEVs, thus leading to the speculation that there was increased neurotoxic protein cargo burden in the morphine dependent macaques. Our in vitro mechanistic findings also showed that the numbers of ADEVs (both 100,000 $\times \mathrm{g}$ and 167,000 $\times \mathrm{g}$ vesicles) from morphine exposed astrocytes were significantly higher than the control group. Previous studies by our group as well as others have shown that morphine can increase the release of $100,000 \times \mathrm{g}$ ADEVs [43], however, this is the first study demonstrating the presence of $167,000 \times \mathrm{g}$ ADEVs also in morphineinduced neuropathogenesis. This $167,000 \times \mathrm{g}$ particles differed in height, diameter and volume as well as protein markers (Argonuate 2 rich) compared with the $100,000 \times$ g ADEVs (Alix, TSG101, CD63 rich). Our findings showed that the $167,000 \times \mathrm{g}$ morphine-ADEVs, but not the $100,000 \times \mathrm{g}$ morphine-ADEVs, had high levels of amyloid and IL- $1 \beta$ cargoes. Interestingly, the release of ADEVs as well as the cargoes, were regulated by HIF- $1 \alpha$ as demonstrated by gene silencing studies. HIF- $1 \alpha$ has been also shown earlier to regulate EV release [87], however, this is the first report demonstrating the regulation of amyloid and neuroinflammatory cargoes in the ADEVs by HIF-1 $\alpha$. ADEVs have been used in the in vitro study, since our study was primarily focused on assessing the role of astrocytes in morphine-induced amyloidosis. For the in vivo study we focused on total brain EVs to validate our hypothesis. We do acknowledge that this does not allow us to dissect the role of EVs from a particular cell type in morphine-induced amyloidosis. Considering the novel role of morphine in amyloidosis, it is important to show the overall effects of morphine on total brain cells and brain cell derived EVs.

In summary, our studies implicate that morphine can induce the expression of transcription factor- HIF- $1 \alpha$, which upon being translocated into the nucleus binds to the BACE 1 promoter to induce its synthesis. Increased level of BACE1 protein and its activity leads to cleavage of the transmembrane protein APP (upregulated independently by morphine) to its toxic $A \beta$ forms, which can cause activation of astrocytes leading to generation of pro-inflammatory cytokines (Fig. 11). Findings by Kalman et. al., showed that morphine administration exhibited a trend of increased membrane bound APP compared with the control rats in the cortex [62]. While the focus of the current study is on astrocytic amyloidosis, the contribution of neuronal amyloidosis cannot be overlooked, however, based on our in vivo data, it appears that the contribution of astrocytes to the process of amyloidosis is higher than that of the non-astrocytic CNS cells. Our findings also demonstrate that the toxic isoforms of amyloids are released by EVs, which, in turn, could be re-distributed and seeded to different sites and cell types within the brain, leading to widespread neurodegeneration and neuroinflammation. Such an amyloid-mediated neurodegeneration as well as neuroinflammation, could contribute to the cognitive deficit observed in long-term opiate users, and could be targeted for future development of adjunctive therapies for chronic opiate users. 


\section{Acknowlegements}

This project has been supported by pilot grant funding from Nebraska Center for Substance Abuse Research (S. Sil) and RO1DA044586, DA040397, DA043138, DA047156, DA050545, MH112848 (S. Buch). We are thankful to Shannon Callen and Shruthi Kumar for their technical assistances. We are also thankful to Drs. Zhiqiang Sun and Yuri L. Lyubchenko, Department of Pharmaceutical Sciences, UNMC for their assistance with the atomic force microscopy. We express our gratitude to Dr. Bhaskar Roy for help with the bioinformatics analysis. We are also thankful to electron microscopy core facility (UNMC).

\section{Conflicts of interest}

The authors disclose no potential conflicts of interest.

\section{Supplementary Materials}

The Supplemenantry data can be found online at: www.aginganddisease.org/EN/10.14336/AD.2021.0406.

\section{References}

[1] Stefano GB, Ptacek R, Kuzelova H, Kream RM (2012). Endogenous morphine: up-to-date review 2011. Folia Biol (Praha), 58:49-56.

[2] Kent CD, Stephens LS, Posner KL, Domino KB (2017). What Adverse Events and Injuries Are Cited in Anesthesia Malpractice Claims for Nonspine Orthopaedic Surgery? Clin Orthop Relat Res, 475:2941-2951.

[3] Friedman H, Eisenstein TK (2004). Neurological basis of drug dependence and its effects on the immune system. J Neuroimmunol, 147:106-108.

[4] Roy S, Wang J, Kelschenbach J, Koodie L, Martin J (2006). Modulation of immune function by morphine: implications for susceptibility to infection. J Neuroimmune Pharmacol, 1:77-89.

[5] Roy S, Barke RA, Loh HH (1998). MU-opioid receptor-knockout mice: role of mu-opioid receptor in morphine mediated immune functions. Brain Res Mol Brain Res, 61:190-194.

[6] Roy S, Ninkovic J, Banerjee S, Charboneau RG, Das S, Dutta R, et al. (2011). Opioid drug abuse and modulation of immune function: consequences in the susceptibility to opportunistic infections. J Neuroimmune Pharmacol, 6:442-465.

[7] Chau DL, Walker V, Pai L, Cho LM (2008). Opiates and elderly: use and side effects. Clin Interv Aging, 3:273-278.

[8] Roy S, Chapin RB, Cain KJ, Charboneau RG, Ramakrishnan S, Barke RA (1997). Morphine inhibits transcriptional activation of IL-2 in mouse thymocytes. Cell Immunol, 179:1-9.
[9] Roy S, Cain KJ, Charboneau RG, Barke RA (1998). Morphine accelerates the progression of sepsis in an experimental sepsis model. Adv Exp Med Biol, 437:2131.

[10] Dinda A, Gitman M, Singhal PC (2005). Immunomodulatory effect of morphine: therapeutic implications. Expert Opin Drug Saf, 4:669-675.

[11] Sullivan MD (2018). Depression Effects on Long-term Prescription Opioid Use, Abuse, and Addiction. Clin J Pain, 34:878-884.

[12] Terrett G, McLennan SN, Henry JD, Biernacki K, Mercuri K, Curran HV, et al. (2014). Prospective memory impairment in long-term opiate users. Psychopharmacology (Berl), 231:2623-2632.

[13] Alam A, Gomes T, Zheng H, Mamdani MM, Juurlink DN, Bell CM (2012). Long-term analgesic use after low-risk surgery: a retrospective cohort study. Arch Intern Med, 172:425-430.

[14] Lawlor PG (2002). The panorama of opioid-related cognitive dysfunction in patients with cancer: a critical literature appraisal. Cancer, 94:1836-1853.

[15] Kamboj SK, Tookman A, Jones L, Curran VH (2005). The effects of immediate-release morphine on cognitive functioning in patients receiving chronic opioid therapy in palliative care. Pain, 117:388-395.

[16] Sjogren P, Christrup LL, Petersen MA, Hojsted J (2005). Neuropsychological assessment of chronic nonmalignant pain patients treated in a multidisciplinary pain centre. Eur J Pain, 9:453-462.

[17] Kinzel S, Weber MS (2016). B Cell-Directed Therapeutics in Multiple Sclerosis: Rationale and Clinical Evidence. CNS Drugs, 30:1137-1148.

[18] Kapogiannis D, Mattson MP (2011). Disrupted energy metabolism and neuronal circuit dysfunction in cognitive impairment and Alzheimer's disease. Lancet Neurol, 10:187-198.

[19] Querfurth HW, LaFerla FM (2010). Alzheimer's disease. N Engl J Med, 362:329-344.

[20] Multhaup G (2006). Amyloid precursor protein and BACE function as oligomers. Neurodegener Dis, 3:270-274.

[21] Vassar R, Bennett BD, Babu-Khan S, Kahn S, Mendiaz EA, Denis P, et al. (1999). Beta-secretase cleavage of Alzheimer's amyloid precursor protein by the transmembrane aspartic protease BACE. Science, 286:735-741.

[22] Vassar R (2002). Beta-secretase (BACE) as a drug target for Alzheimer's disease. Adv Drug Deliv Rev, 54:1589-1602.

[23] Roggo S (2002). Inhibition of BACE, a promising approach to Alzheimer's disease therapy. Curr Top Med Chem, 2:359-370.

[24] Anthony IC, Norrby KE, Dingwall T, Carnie FW, Millar T, Arango JC, et al. (2010). Predisposition to accelerated Alzheimer-related changes in the brains of human immunodeficiency virus negative opiate abusers. Brain, 133:3685-3698.

[25] Ramage SN, Anthony IC, Carnie FW, Busuttil A, Robertson R, Bell JE (2005). Hyperphosphorylated tau and amyloid precursor protein deposition is increased 
in the brains of young drug abusers. Neuropathol Appl Neurobiol, 31:439-448.

[26] Sil S, Hu G, Liao K, Niu F, Callen S, Periyasamy P, et al. (2020). HIV-1 Tat-mediated astrocytic amyloidosis involves the HIF-1alpha/lncRNA BACE1-AS axis. PLoS Biol, 18:e3000660.

[27] Cai Y, Yang L, Hu G, Chen X, Niu F, Yuan L, et al. (2016). Regulation of morphine-induced synaptic alterations: Role of oxidative stress, ER stress, and autophagy. J Cell Biol, 215:245-258.

[28] Gonzalez-Reyes RE, Nava-Mesa MO, Vargas-Sanchez K, Ariza-Salamanca D, Mora-Munoz L (2017). Involvement of Astrocytes in Alzheimer's Disease from a Neuroinflammatory and Oxidative Stress Perspective. Front Mol Neurosci, 10:427.

[29] Garwood CJ, Pooler AM, Atherton J, Hanger DP, Noble W (2011). Astrocytes are important mediators of Abeta-induced neurotoxicity and tau phosphorylation in primary culture. Cell Death Dis, 2:e167.

[30] Margolis L, Sadovsky Y (2019). The biology of extracellular vesicles: The known unknowns. PLoS Biol, 17:e3000363.

[31] Gupta A, Pulliam L (2014). Exosomes as mediators of neuroinflammation. J Neuroinflammation, 11:68.

[32] Thery C, Ostrowski M, Segura E (2009). Membrane vesicles as conveyors of immune responses. Nat Rev Immunol, 9:581-593.

[33] Cocucci E, Racchetti G, Meldolesi J (2009). Shedding microvesicles: artefacts no more. Trends Cell Biol, 19:43-51.

[34] Piccin A, Murphy WG, Smith OP (2007). Circulating microparticles: pathophysiology and clinical implications. Blood Rev, 21:157-171.

[35] Shi Q, Hodara V, Meng Q, Voruganti VS, Rice K, Michalek JE, et al. (2014). Early endothelial damage detected by circulating particles in baboons fed a diet high in simple carbohydrates in conjunction with saturated or unsaturated fat. Am J Cardiovasc Dis, 4:123-132.

[36] Taylor DD, Gercel-Taylor C (2011). Exosomes/microvesicles: mediators of cancerassociated immunosuppressive microenvironments. Semin Immunopathol, 33:441-454.

[37] Rabinowits G, Gercel-Taylor C, Day JM, Taylor DD, Kloecker GH (2009). Exosomal microRNA: a diagnostic marker for lung cancer. Clin Lung Cancer, 10:42-46.

[38] Narayanan A, Iordanskiy S, Das R, Van Duyne R, Santos S, Jaworski E, et al. (2013). Exosomes derived from HIV-1-infected cells contain trans-activation response element RNA. J Biol Chem, 288:2001420033.

[39] Sardar Sinha M, Ansell-Schultz A, Civitelli L, Hildesjo C, Larsson M, Lannfelt L, et al. (2018). Alzheimer's disease pathology propagation by exosomes containing toxic amyloid-beta oligomers. Acta Neuropathol, 136:41-56.

[40] Shi M, Liu C, Cook TJ, Bullock KM, Zhao Y, Ginghina C, et al. (2014). Plasma exosomal alpha-synuclein is likely CNS-derived and increased in Parkinson's disease. Acta Neuropathol, 128:639-650.

[41] Roy J, Saucier D, O'Connell C, Morin PJ (2019). Extracellular vesicles and their diagnostic potential in amyotrophic lateral sclerosis. Clin Chim Acta, 497:2734.

[42] Sampey GC, Saifuddin M, Schwab A, Barclay R, Punya S, Chung MC, et al. (2016). Exosomes from HIV-1infected Cells Stimulate Production of Proinflammatory Cytokines through Trans-activating Response (TAR) RNA. J Biol Chem, 291:1251-1266.

[43] Hu G, Liao K, Niu F, Yang L, Dallon BW, Callen S, et al. (2018). Astrocyte EV-Induced lincRNA-Cox2 Regulates Microglial Phagocytosis: Implications for Morphine-Mediated Neurodegeneration. Mol Ther Nucleic Acids, 13:450-463.

[44] Yang L, Niu F, Yao H, Liao K, Chen X, Kook Y, et al. (2018). Exosomal miR-9 Released from HIV Tat Stimulated Astrocytes Mediates Microglial Migration. J Neuroimmune Pharmacol, 13:330-344.

[45] Yuyama K, Igarashi Y (2017). Exosomes as Carriers of Alzheimer's Amyloid-ss. Front Neurosci, 11:229.

[46] Beretta C, Nikitidou E, Streubel-Gallasch L, Ingelsson M, Sehlin D, Erlandsson A (2020). Extracellular vesicles from amyloid-beta exposed cell cultures induce severe dysfunction in cortical neurons. Sci Rep, 10:19656.

[47] Eitan E, Hutchison ER, Marosi K, Comotto J, Mustapic M, Nigam SM, et al. (2016). Extracellular VesicleAssociated Abeta Mediates Trans-Neuronal Bioenergetic and $\mathrm{Ca}(2+)$-Handling Deficits in Alzheimer's Disease Models. NPJ Aging Mech Dis, 2.

[48] Lee S, Mankhong S, Kang JH (2019). Extracellular Vesicle as a Source of Alzheimer's Biomarkers: Opportunities and Challenges. Int J Mol Sci, 20.

[49] Watson LS, Hamlett ED, Stone TD, Sims-Robinson C (2019). Neuronally derived extracellular vesicles: an emerging tool for understanding Alzheimer's disease. Mol Neurodegener, 14:22.

[50] Sil S, Periyasamy P, Guo ML, Callen S, Buch S (2018). Morphine-Mediated Brain Region-Specific Astrocytosis Involves the ER Stress-Autophagy Axis. Mol Neurobiol, 55:6713-6733.

[51] Periyasamy P, Thangaraj A, Guo ML, Hu G, Callen S, Buch S (2018). Epigenetic Promoter DNA Methylation of miR-124 Promotes HIV-1 Tat-Mediated Microglial Activation via MECP2-STAT3 Axis. J Neurosci, 38:5367-5383.

[52] Goto Y, Zeng L, Yeom CJ, Zhu Y, Morinibu A, Shinomiya K, et al. (2015). UCHL1 provides diagnostic and antimetastatic strategies due to its deubiquitinating effect on HIF-1alpha. Nat Commun, 6:6153.

[53] Zhang Q, Higginbotham JN, Jeppesen DK, Yang YP, Li W, McKinley ET, et al. (2019). Transfer of Functional Cargo in Exomeres. Cell Rep, 27:940-954 e946.

[54] Dragovic RA, Gardiner C, Brooks AS, Tannetta DS, Ferguson DJ, Hole P, et al. (2011). Sizing and phenotyping of cellular vesicles using Nanoparticle Tracking Analysis. Nanomedicine, 7:780-788. 
[55] Hurwitz SN, Sun L, Cole KY, Ford CR, 3rd, Olcese JM, Meckes DG, Jr. (2018). An optimized method for enrichment of whole brain-derived extracellular vesicles reveals insight into neurodegenerative processes in a mouse model of Alzheimer's disease. $\mathbf{J}$ Neurosci Methods, 307:210-220.

[56] Lyubchenko YL, Shlyakhtenko LS, Ando T (2011). Imaging of nucleic acids with atomic force microscopy. Methods, 54:274-283.

[57] Han J, Li L, Zhang Z, Xiao Y, Lin J, Zheng L, et al. (2007). Platelet-derived growth factor C plays a role in the branchial arch malformations induced by retinoic acid. Birth Defects Res A Clin Mol Teratol, 79:221230.

[58] Pare EM, Monforte JR, Thibert RJ (1984). Morphine concentrations in brain tissue from heroin-associated deaths. J Anal Toxicol, 8:213-216.

[59] Liao K, Niu F, Hu G, Yang L, Dallon B, Villarreal D, et al. (2020). Morphine-mediated release of miR-138 in astrocyte-derived extracellular vesicles promotes microglial activation. J Extracell Vesicles, 10:e12027.

[60] $\mathrm{Hu}$ G, Gong AY, Roth AL, Huang BQ, Ward HD, Zhu $\mathrm{G}$, et al. (2013). Release of luminal exosomes contributes to TLR4-mediated epithelial antimicrobial defense. PLoS Pathog, 9:e1003261.

[61] Hu G, Yao H, Chaudhuri AD, Duan M, Yelamanchili SV, Wen H, et al. (2012). Exosome-mediated shuttling of microRNA-29 regulates HIV Tat and morphinemediated neuronal dysfunction. Cell Death Dis, 3:e381.

[62] Kalman J, Bjelik A, Hugyecz M, Timar J, Gyarmati Z, Zana M, et al. (2007). 3,4Methylenedioxymethamphetamine (MDMA), but not morphine, alters APP processing in the rat brain. Int $\mathbf{J}$ Neuropsychopharmacol, 10:183-190.

[63] Bryant CD, Roberts KW, Byun JS, Fanselow MS, Evans CJ (2006). Morphine analgesic tolerance in 129P3/J and 129S6/SvEv mice. Pharmacol Biochem Behav, 85:769-779.

[64] Volterra A, Meldolesi J (2005). Astrocytes, from brain glue to communication elements: the revolution continues. Nat Rev Neurosci, 6:626-640.

[65] Colomar A, Robitaille R (2004). Glial modulation of synaptic transmission at the neuromuscular junction. Glia, 47:284-289.

[66] Bachoo RM, Kim RS, Ligon KL, Maher EA, Brennan C, Billings N, et al. (2004). Molecular diversity of astrocytes with implications for neurological disorders. Proc Natl Acad Sci U S A, 101:8384-8389.

[67] Vasile F, Dossi E, Rouach N (2017). Human astrocytes: structure and functions in the healthy brain. Brain Struct Funct, 222:2017-2029.

[68] Foerde K, Shohamy D (2011). The role of the basal ganglia in learning and memory: insight from Parkinson's disease. Neurobiol Learn Mem, 96:624636.

[69] Elhalal A, Davelaar EJ, Usher M (2014). The role of the frontal cortex in memory: an investigation of the Von Restorff effect. Front Hum Neurosci, 8:410.
[70] Gremel CM, Lovinger DM (2017). Associative and sensorimotor cortico-basal ganglia circuit roles in effects of abused drugs. Genes Brain Behav, 16:71-85.

[71] Schmidt A, Denier N, Magon S, Radue EW, Huber CG, Riecher-Rossler A, et al. (2015). Increased functional connectivity in the resting-state basal ganglia network after acute heroin substitution. Transl Psychiatry, 5:e533.

[72] Baldo BA (2016). Prefrontal Cortical Opioids and Dysregulated Motivation: A Network Hypothesis. Trends Neurosci, 39:366-377.

[73] Saido TC, Yokota M, Maruyama K, Yamao-Harigaya W, Tani E, Ihara Y, et al. (1994). Spatial resolution of the primary beta-amyloidogenic process induced in postischemic hippocampus. J Biol Chem, 269:1525315257.

[74] Reijmer YD, van Veluw SJ, Greenberg SM (2016). Ischemic brain injury in cerebral amyloid angiopathy. $\mathrm{J}$ Cereb Blood Flow Metab, 36:40-54.

[75] Spangenberg EE, Lee RJ, Najafi AR, Rice RA, Elmore MR, Blurton-Jones M, et al. (2016). Eliminating microglia in Alzheimer's mice prevents neuronal loss without modulating amyloid-beta pathology. Brain, 139:1265-1281.

[76] Koodie L, Ramakrishnan S, Roy S (2010). Morphine suppresses tumor angiogenesis through a HIF1alpha/p38MAPK pathway. Am J Pathol, 177:984-997.

[77] Roy S, Balasubramanian S, Wang J, Chandrashekhar Y, Charboneau R, Barke R (2003). Morphine inhibits VEGF expression in myocardial ischemia. Surgery, 134:336-344.

[78] Minter MR, Taylor JM, Crack PJ (2016). The contribution of neuroinflammation to amyloid toxicity in Alzheimer's disease. J Neurochem, 136:457-474.

[79] Cisternas P, Taylor X, Lasagna-Reeves CA (2019). The Amyloid-Tau-Neuroinflammation Axis in the Context of Cerebral Amyloid Angiopathy. Int J Mol Sci, 20.

[80] Welikovitch LA, Do Carmo S, Magloczky Z, Malcolm JC, Loke J, Klein WL, et al. (2020). Early intraneuronal amyloid triggers neuron-derived inflammatory signaling in APP transgenic rats and human brain. Proc Natl Acad Sci U S A, 117:6844-6854.

[81] Kalra H, Drummen GP, Mathivanan S (2016). Focus on Extracellular Vesicles: Introducing the Next Small Big Thing. Int J Mol Sci, 17:170.

[82] Sofroniew MV, Vinters HV (2010). Astrocytes: biology and pathology. Acta Neuropathol, 119:7-35.

[83] Yanez-Mo M, Siljander PR, Andreu Z, Zavec AB, Borras FE, Buzas EI, et al. (2015). Biological properties of extracellular vesicles and their physiological functions. J Extracell Vesicles, 4:27066.

[84] van den Boorn JG, Schlee M, Coch C, Hartmann G (2011). SiRNA delivery with exosome nanoparticles. Nat Biotechnol, 29:325-326.

[85] Badhwar A, Haqqani AS (2020). Biomarker potential of brain-secreted extracellular vesicles in blood in Alzheimer's disease. Alzheimers Dement (Amst), 12:e12001.

[86] Huttunen HJ, Puglielli L, Ellis BC, MacKenzie Ingano LA, Kovacs DM (2009). Novel N-terminal cleavage of 
APP precludes Abeta generation in ACAT-defective AC29 cells. J Mol Neurosci, 37:6-15.

[87] Zhang W, Zhou X, Yao Q, Liu Y, Zhang H, Dong Z (2017). HIF-1-mediated production of exosomes during hypoxia is protective in renal tubular cells. Am J Physiol Renal Physiol, 313:F906-F913. 